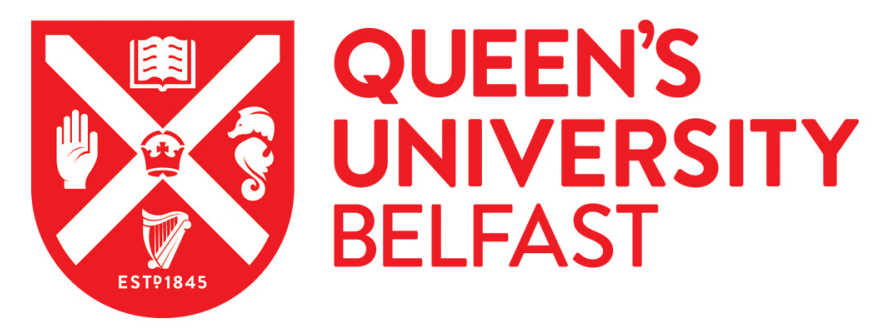

\title{
Standardising RNA profiling based biomarker application in cancer - the need for robust control of technical variables
}

\author{
Stewart, J. P., Richman, S., Maughan, T., Lawler, M., Dunne, P. D., \& Salto-Tellez, M. (2017). Standardising \\ RNA profiling based biomarker application in cancer - the need for robust control of technical variables. \\ Biochimica et biophysica acta. https://doi.org/10.1016/j.bbcan.2017.05.005
}

Published in:

Biochimica et biophysica acta

Document Version:

Peer reviewed version

Queen's University Belfast - Research Portal:

Link to publication record in Queen's University Belfast Research Portal

Publisher rights

() 2017 Elsevier B.V. All rights reserved. This manuscript is distributed under a Creative Commons Attribution-NonCommercial-NoDerivs License (https://creativecommons.org/licenses/by-nc-nd/4.0/), which permits distribution and reproduction for non-commercial purposes, provided the author and source are cited.

\section{General rights}

Copyright for the publications made accessible via the Queen's University Belfast Research Portal is retained by the author(s) and / or other copyright owners and it is a condition of accessing these publications that users recognise and abide by the legal requirements associated with these rights.

Take down policy

The Research Portal is Queen's institutional repository that provides access to Queen's research output. Every effort has been made to ensure that content in the Research Portal does not infringe any person's rights, or applicable UK laws. If you discover content in the Research Portal that you believe breaches copyright or violates any law, please contact openaccess@qub.ac.uk. 


\section{Accepted Manuscript}

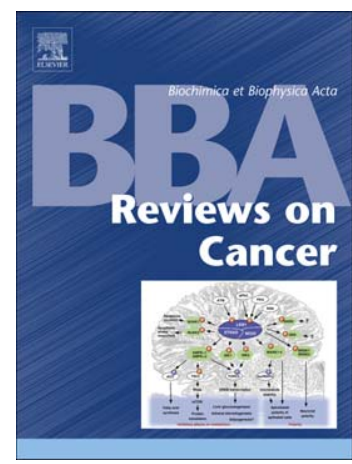

Standardising RNA profiling based biomarker application in cancer - the need for robust control of technical variables

James P. Stewart, Susan Richman, Tim Maughan, Mark Lawler, Philip D. Dunne, Manuel Salto-Tellez
PII:
S0304-419X(17)30004-5
DOI:
doi: 10.1016/j.bbcan.2017.05.005
Reference:
BBACAN 88161

To appear in:

BBA - Reviews on Cancer

Received date:

2 February 2017

Revised date:

21 May 2017

Accepted date:

22 May 2017

Please cite this article as: James P. Stewart, Susan Richman, Tim Maughan, Mark Lawler, Philip D. Dunne, Manuel Salto-Tellez, Standardising RNA profiling based biomarker application in cancer - the need for robust control of technical variables, $B B A$ - Reviews on Cancer (2017), doi:10.1016/j.bbcan.2017.05.005

This is a PDF file of an unedited manuscript that has been accepted for publication. As a service to our customers we are providing this early version of the manuscript. The manuscript will undergo copyediting, typesetting, and review of the resulting proof before it is published in its final form. Please note that during the production process errors may be discovered which could affect the content, and all legal disclaimers that apply to the journal pertain. 
Standardising RNA profiling based biomarker application in cancer - the need for robust control of technical variables

James P. Stewart ${ }^{1,2}$, Susan Richman ${ }^{3}$, Tim Maughan ${ }^{4}$, Mark Lawler ${ }^{1}$, Philip D. Dunne ${ }^{1, *}$ and Manuel Salto-Tellez ${ }^{1,2, *, \wedge}$

${ }^{1}$ Centre for Cancer Research and Cell Biology, Queen’s University Belfast, UK

${ }^{2}$ Northern Ireland Molecular Pathology Laboratory, Queen's University Belfast, UK

${ }^{3}$ Department of Pathology and Tumour Biology, St James University Hospital, Leeds, UK.

${ }^{4}$ CRUK/MRC Oxford Institute for Radiation Oncology, University of Oxford, UK

*Joint Senior Authors

${ }^{\wedge}$ Corresponding Author

\section{Corresponding Author Address:}

Centre for Cancer Research \& Cell Biology, Queens University Belfast, 97 Lisburn Road, Belfast, BT9 7BL.Email: m.salto-tellez@ qub.ac.uk 


\section{$\underline{\text { Abstract }}$}

Histopathology-based staging of colorectal cancer (CRC) has utility in assessing the prognosis of patient subtypes, but as yet cannot accurately predict individual patient's treatment response. Transcriptomics approaches, using array based or next generation sequencing (NGS) platforms, of formalin fixed paraffin embedded tissue can be harnessed to develop multi-gene biomarkers for predicting both prognosis and treatment response, leading to stratification of treatment. While transcriptomics can shape future biomarker development, currently $<1 \%$ of published biomarkers become clinically validated tests, often due to poor study design or lack of independent validation. In this review of a large number of CRC transcriptional studies, we identify recurrent sources of technical variability that encompass collection, preservation and storage of malignant tissue, nucleic acid extraction, methods to quantitate RNA transcripts and data analysis pipelines. We propose a series of defined steps for removal of these confounding issues, to ultimately aid in the development of more robust clinical biomarkers.

Keywords: Microarray, RNA profiling, NGS, transcriptome, FFPE, biomarker

Abbreviations: CRC, colorectal cancer; AJCC, American Joint Committee on Cancer; TNM, Tumour Node Metastasis; GEP, Gene expression profile; CRCSC, CRC Subtyping Consortium; CMS, consensus molecular subgroups; MSI, microsatellite instability; FFPE,

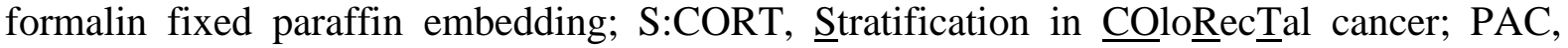
probably approximately correct; SOP, standard operating procedures; FF, fresh frozen; IVT, in vitro transcription; RIN, RNA integrity number; BBRB, Biorepositories and Biospecimen Research Branch; CDP, Cancer Diagnosis Program; TCGA, The Cancer Genome Atlas; 
ERCC, External RNA Controls Consortium; LDT, laboratory developed test; CLIA, Clinical Laboratory Improvement Amendments.

\section{Variability in colorectal cancer transcriptomic studies}

Colorectal cancer (CRC) is the United Kingdom's second most common cause of cancer deaths ${ }^{1}$. Diagnostic staging of CRC relies on the American Joint Committee on Cancer (AJCC) Tumour Node Metastasis (TNM) staging system ${ }^{2}$. Classification into AJCC staging groups is based on extent of local tumour invasion, regional lymph node involvement and evidence of distant metastasis. While this staging system has intrinsic prognostic value, this initial categorisation is insufficient for predicting outcome following treatment ${ }^{3}$. Several clinical and histopathological markers have been reported to be 'prognosis predictors' for Stage II patients, by indicating benefit from adjuvant chemotherapy. These include extramural vascular invasion, grade 3/poor differentiation, serum carcinoembryonic antigen levels, T4 stage/perforation, perineural invasion, $C D X 2$, obstructive tumours, mucinous tumours, tumour budding and microenvironmental factors such as immune cell infiltration ${ }^{1,3-}$ 9 .

Biomarker development aimed at guiding clinical decision-making for adjuvant therapy has been undermined by a lack of translation to clinical applicability, leading to a high attrition rate in effective cancer biomarkers. This is due in part to confounding issues such as inadequate study design, poorly selected clinical cohorts, technically inadequate assays, invalidated technology, inappropriate statistical analysis or lack of clinical relevance ${ }^{10}$. Thus, additional molecular-based biomarkers, informed by underlying CRC biology and the contributing role of the microenvironment, are required to stratify patients for biologyinformed treatment. 


\section{Comparison of single and multi-gene diagnostic testing: The EGFR paradigm}

Molecular DNA-based biomarker testing, traditionally involving analysis of a single gene/mutation, have made important contributions in modern therapeutic decision-making in CRC, but may also have certain limitations. An example of the limitations associated with low throughput-gene testing is evident from the patient stratification approach used for cetuximab and panitumumab, monoclonal antibodies which target non-identical epitopes on the extracellular domain of $E G F R^{11}$. Screening for mutations in KRAS exon 2 (codon 12/13) was initially employed to predict lack of response to anti-EGFR therapeutics, but recently studies postulate potential greater clinical utility in assessing additional mutations in KRAS exon 2, 3, and 4 and NRAS exons 2, 3, and 4, PIK3CA and BRAF mutations and HER2 amplification ${ }^{12-14}$. Multi-gene assays, which encompass screening of at least NRAS, KRAS, PIK3CA and BRAF mutations, could enable better characterisation and ultimately more precise targeting of several druggable EGFR pathway components using therapeutic combinations to circumvent chemoresistance ${ }^{15}$. Transcriptional outlier analysis can be used to identify high expression of mRNA transcripts in individual samples, in comparison to the remainder of the cohort, which encode proteins that can be therapeutically targeted enabling individualised treatment options. Transcriptional outlier analysis of cetuximab resistant CRC cell lines, which were wild-type for KRAS, NRAS and BRAF has enabled the identification of tyrosine kinase that can be therapeutically targeted to overcome cetuximab resistance ${ }^{16}$.

\section{Requirement for a common approach to transcriptomic analysis of clinical specimens}

In recent years, numerous efforts to precisely define the molecular landscape of CRC using transcriptional array-based studies have enabled classification of patients into 3-6 subgroups based on their underlying gene expression ${ }^{17-22}$. Recently, a CRC Subtyping Consortium (CRCSC) established a consensus molecular CRC classification comprising four subgroups 
(CMS1-CMS4) based on gene expression profiling (GEP) data from six key molecular taxonomy papers ${ }^{23}$. The CRCSC classifier demonstrated a robust performance across GEP platforms and sample collections, although the clinical value of prospective patient classification according to CMS subgroup for treatment selection remains to be tested. Importantly, while each study has identified prognostic biology associated with their identified subtypes, with the exception of the microsatellite instability (MSI) subtype (CMS1) and another subtype defined by high expression of mesenchymal genes (CMS4) ${ }^{23}$ there are very few similarities between biological characteristics of the biomarker-driven classifiers that select these subgroups. On detailed examination, of the six classification signatures used in the CRCSC study, we identified only one gene, Quinolinate phosphoribosyltransferase $(Q P R T)$, present in all signatures ${ }^{24}$ (Figure 1). The protein encoded by the $Q P R T$ gene is involved in de novo NAD biosynthesis using quinolinic acid ${ }^{25}$. The lack of common genes in different molecular, predictive or prognostic classifiers is often due to a combination of (i) small training sets, (ii) cohorts being limited to one institution or (iii) samples from patients with differing percentages of AJCC stages being used in signature generation, resulting in low reproducibility in independent datasets ${ }^{26,27}$. 


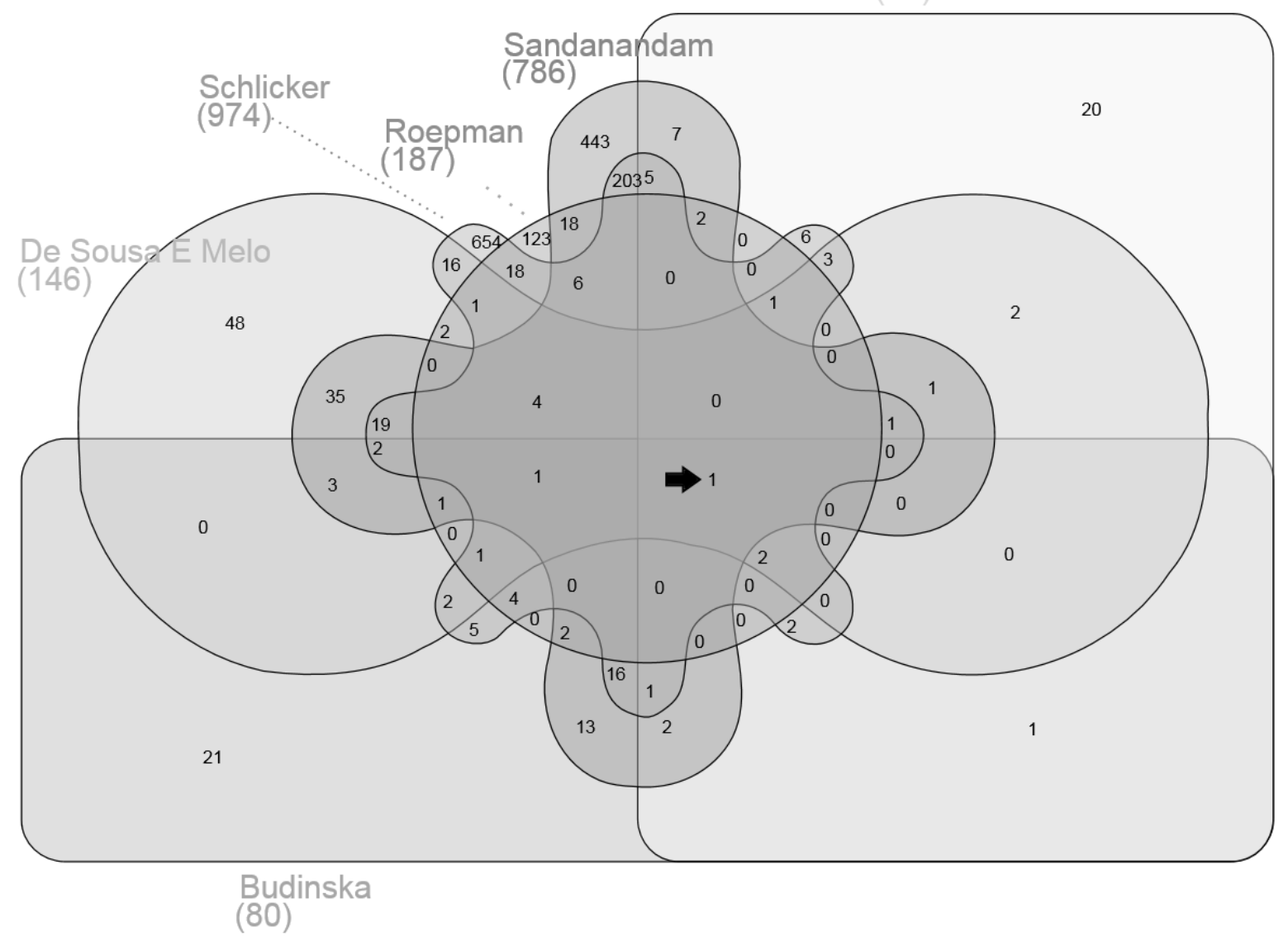

Figure 1. Lack of commonality between molecular taxonomy classification signatures. A

Venn diagram, using InteractiVenn, was created, incorporating six published molecular taxonomy classification signatures used to create the CMS classification system ${ }^{24}$. Only one gene, highlighted by the black arrow, encoding Quinolinate Phosphoribosyltransferase was shown to be common among all six signatures.

The lack of commonality that we have highlighted between gene expression signatures may potentially be one of the reasons why multi-gene biomarker development rarely translates to a validated clinical test. Therefore, there is a requirement to examine the multi-gene biomarker development pathway to identify study design issues or technical variability that compromise robust gene expression signatures development for stratification of patients by prognosis or response to treatment. The multi-step biomarker validation pathway involves 
assessment of; (1) availability and quality of clinical specimens, (2) transcriptomic assay performance characteristics, (3) data pre-processing algorithms, (4) mathematical predictor model development (5) and assessment of its performance, (6) clinical interpretation of the test result, (7) clinical trial design, (8) ethical, legal, and regulatory issues ${ }^{28}$.

In this review, we focus on the early steps of this biomarker validation pathway and examine in detail the level of scrutiny an investigator, looking to initiate a transcriptomic profiling study, should achieve to negate the introduction of study and/or technical variability. To help the reader understand the complexity involved in transcriptome profiling studies, we have highlighted the different methods published by independent CRC transcriptome profiling studies $^{29-63}$. In the online supplementary table 1 , we provide a summary of 57 transcriptome profiling studies and detail relevant information regarding the study cohorts such as small cohort sizes, different proportions of patients with stages 1-4 and number of study sites. We also highlighted that groups have differences in the level of tumour content they found acceptable for the study and the way in which the tissue was preserved. There are two common tissue preservation methods currently used for GEP studies; fresh frozen (FF) and formalin fixed paraffin embedding (FFPE), with FFPE almost universally used, due to retention of morphological features. FFPE is a well-established preservation method for histopathological assessment but results in extensive RNA fragmentation and cross-linking, impacting on high quality GEP. It is advantageous if GEP studies can address the RNA fragmentation and cross-linking issues, thus enabling FFPE samples to be utilised, as it is estimated that a billion FFPE samples are archived in hospitals and tissue banks, often with substantial clinical follow up ${ }^{64}$. 
Aside from variables related to collection, preservation and storage of the tissue we noted that investigators often used different clinical endpoints to define good and poor prognosis which could potentially confound validating gene expression signatures on independent cohorts. To complicate matters, investigators have employed different RNA extraction protocols and microarray platforms from a number of different vendors (Affymetrix, Illumina, Agilent, Almac) to perform the quantitation of the RNA transcripts and the probe sets utilised by different array manufacturers can differ in size and also the target region they anneal to. Therefore, it is not uncommon to find in some validation studies that expression data derived from specific probe sets is often omitted when comparing a gene signature established using one microarray platform with gene expression data derived from a different vendor's platform. Of note, published studies utilised different pre-processing algorithms such as RMA, MAS 5.0, iterPLIER and other vendor specific packages which can affect the outcome of whether a gene is included or excluded in a multi-gene biomarker. We also detail the number of studies which do not conduct an independent validation of their developed gene expression signature. In Table $1 \mathrm{~A}-\mathrm{C}$, we provide a synopsis of the online supplementary data to highlight the technical variability that occurs within multi-gene signature based biomarker CRC studies aimed at defining molecular taxonomy ${ }^{17-22}$, patient prognosis ${ }^{6,13,65-73}$ or treatment response ${ }^{74-78}$. Standardisation of these studies was commonly confounded by an incomplete control of pre-analytical variables, variable performance on different highthroughput technologies, use of an assortment of bioinformatics curation approaches. Additionally, the resulting multi-gene signatures were often not subjected to validation on an independent cohort of patient samples. While the studies detailed in Tables 1A-C primarily focus on array-based studies, several reports in the last 3-4 years have begun to describe the quantitation of RNA transcripts using a next generation sequencing approach, (RNA-seq), on FF resected tissue from CRC patients ${ }^{79-82}$. 
This review identifies sources of technical variability in the GEP array-based biomarker development pipeline using FFPE CRC tumours. We categorise pre-analytical and analytical variables into nine distinct sections which can confound development and validation of published molecular signatures, to aid in the standardisation of the pipeline. This standardised approach is currently being implemented in the transcriptional profiling of 2000 CRC FFPE samples within the Medical Research Council and Cancer Research UK funded Stratification in COloRecTal cancer (S:CORT) consortium ${ }^{83}$. We also detail how eight of the nine categories of technical variability that we identify, also have application to quantitation of RNA transcripts using a NGS RNA-seq approach. 


\section{Table 1A Molecular Classification Studies}

\begin{tabular}{|c|c|c|c|c|c|c|c|c|c|c|c|c|c|c|c|c|c|c|c|c|c|c|}
\hline \multirow[b]{2}{*}{ 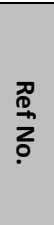 } & \multicolumn{9}{|c|}{ Patient Cohort Information } & \multicolumn{3}{|c|}{ Sample Details } & \multicolumn{2}{|c|}{ RNA Profiling Objectives } & \multicolumn{3}{|c|}{ Platform and method to derive multi-gene signature } & \multicolumn{5}{|c|}{ Validation Group Within Original Paper } \\
\hline & 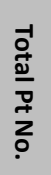 & 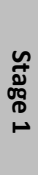 & 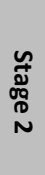 & 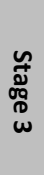 & 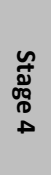 & $\begin{array}{l}\frac{0}{3} \\
\frac{2}{0} \\
\frac{0}{0} \\
\frac{1}{2}\end{array}$ & 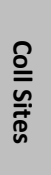 & 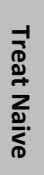 & 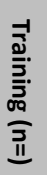 & 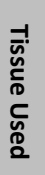 & $\begin{array}{l}\frac{1}{5} \\
\stackrel{0}{a} \\
\frac{0}{9}\end{array}$ & 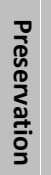 & 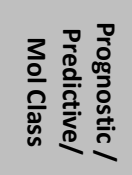 & 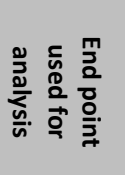 & $\begin{array}{l}\frac{0}{2} \\
\stackrel{0}{0} \frac{1}{3} \\
\frac{3}{3} \\
\frac{3}{2}\end{array}$ & 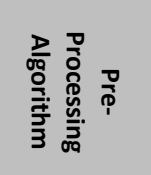 & 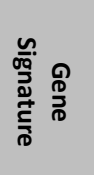 & 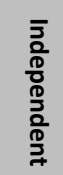 & $\begin{array}{l}\frac{2}{3} \\
\frac{0}{2} \\
\frac{1}{3} \\
\text { II }\end{array}$ & $\frac{\stackrel{2}{2}}{\stackrel{2}{\circ}}$ & 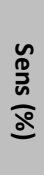 & $\begin{array}{l}\frac{n}{0} \\
\frac{0}{0} \\
\text { do }\end{array}$ \\
\hline$\tilde{\omega}$ & 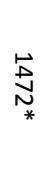 & $\stackrel{\infty}{\sim}$ & 官 & $\underset{\infty}{\omega}$ & $\stackrel{\vec{\omega}}{\omega}$ & $\circ$ & $\begin{array}{l}\frac{3}{\frac{2}{0}} \\
\frac{\overline{\bar{t}}}{\overline{0}}\end{array}$ & 吊 & $\stackrel{\vec{b}}{\omega}$ & $\dashv$ & $\bar{z}_{0}$ & ᄁᄁ & 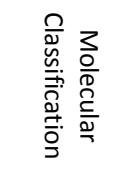 & $\frac{Z}{s}$ & 总 & $\frac{\pi}{3}$ & u & 同 & $\stackrel{\stackrel{E}{N}}{N}$ & z & z & $z$ \\
\hline$\tilde{n}$ & 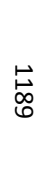 & $\sum_{\mathrm{z}}$ & $\bar{z}_{0}$ & $\sum_{\mathrm{z}}$ & $\bar{z}_{\mathrm{v}}$ & $\sum_{i}$ & $\begin{array}{l}\frac{3}{c} \\
\frac{\overline{0}}{\overline{0}} \\
\frac{\overline{0}}{D}\end{array}$ & $\sum_{i}$ & 点 & $\dashv$ & $\bar{z}_{\mathbf{v}}$ & $\sum_{\mathrm{z}}$ & 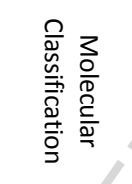 & & 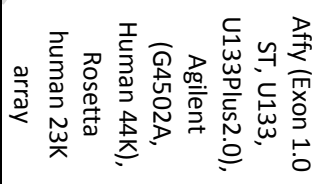 & 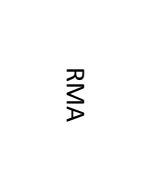 & $\stackrel{\infty}{\infty}$ & 同 & $\stackrel{\vec{f}}{f}$ & z & z & $z$ \\
\hline$\tilde{n}$ & $\begin{array}{c}\vec{b} \\
\stackrel{\infty}{y}\end{array}$ & $\sum_{\mathrm{z}}$ & $\sum_{0}$ & $\sum_{\mathrm{j}}$ & $\bar{z}_{v}$ & $\sum_{i}$ & 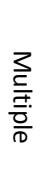 & $\sum_{j}$ & ๕ & $\dashv$ & $\sum_{j}$ & $\bar{z}_{\mathbf{z}}$ & 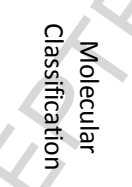 & $\underset{D}{Z}$ & 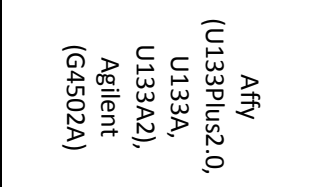 & 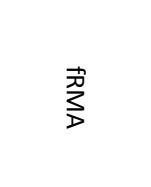 & 奌 & 同 & $\stackrel{\leftrightarrow}{\sigma}$ & z & z & z \\
\hline$\approx$ & 峁 & $\bar{z}_{\mathrm{z}}$ & $\bar{z}_{\mathrm{j}}$ & $\bar{z}_{\mathrm{j}}$ & $\bar{z}_{\mathrm{j}}$ & $\bar{z}_{\mathbf{z}}$ & 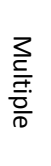 & $\bar{z}_{0}$ & 只 & $\dashv$ & $\sum_{j}$ & $\bar{z}_{0}$ & 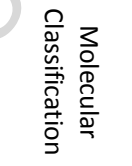 & $\frac{Z}{D}$ & 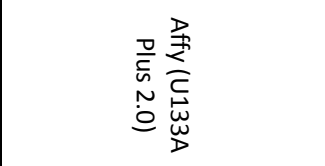 & $\underset{D}{\frac{\pi}{b}}$ & 点 & ঐ & 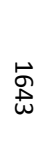 & z & z & z \\
\hline$\tilde{N}$ & $\underset{\sim}{\sim}$ & $\bar{z}$ & $\bar{z}_{i}$ & $\sum_{i}$ & $\bar{z}_{\mathrm{j}}$ & $\bar{z}_{\mathbf{z}}$ & $\begin{array}{l}\frac{3}{\frac{2}{\bar{D}}} \\
\frac{\overline{\bar{t}}}{\overline{0}}\end{array}$ & $\bar{z}_{0}$ & $\stackrel{\bullet}{v}$ & $\begin{array}{l}2 \\
3 \\
\pm \\
-1\end{array}$ & $\bar{z}$ & 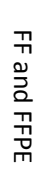 & 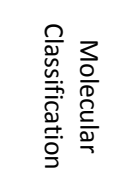 & $\underset{D}{Z}$ & 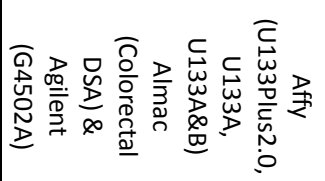 & 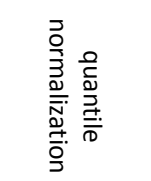 & $\stackrel{\infty}{\omega}$ & 同 & 莒 & z & $z$ & z \\
\hline$\tilde{A}$ & $\underset{\omega}{\tilde{\omega}}$ & $\tilde{A}$ & 苂 & $\tilde{U}$ & $\infty$ & 0 & 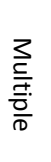 & $\bar{z}_{0}$ & $\begin{array}{l}\vec{\infty} \\
\infty\end{array}$ & $\dashv$ & $\bar{z}$ & ᄁᄁ & 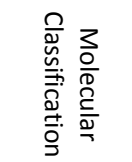 & $\underset{s}{Z}$ & 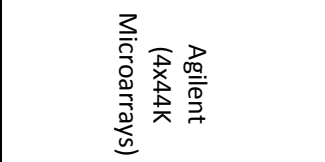 & 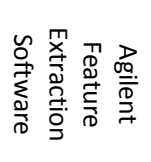 & $\stackrel{\infty}{b^{\prime}}$ & 冏 & $\underset{\omega}{\stackrel{W}{\omega}}$ & z & $z$ & z \\
\hline
\end{tabular}




\section{Table 1B Predictive Multi-gene Signature Studies}

\begin{tabular}{|c|c|c|c|c|c|c|c|c|c|c|c|c|c|c|c|c|c|c|c|c|c|c|}
\hline \multirow[b]{2}{*}{$\begin{array}{l}\mathbb{D}_{0} \\
\stackrel{\mathbb{2}}{\mathbf{z}} \\
\end{array}$} & \multicolumn{9}{|c|}{ Patient Cohort Information } & \multicolumn{3}{|c|}{ Sample Details } & \multicolumn{2}{|c|}{ RNA Profiling Objectives } & \multicolumn{3}{|c|}{ Platform and method to derive multi-gene signature } & \multicolumn{5}{|c|}{ Validation Group Within Original Paper } \\
\hline & 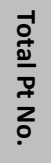 & 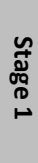 & 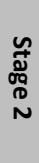 & 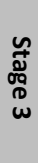 & 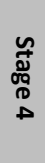 & $\frac{9}{2}$ & 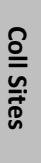 & 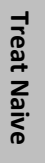 & 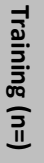 & 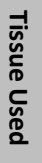 & 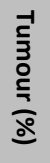 & 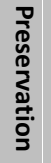 & 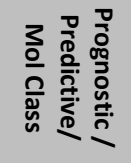 & 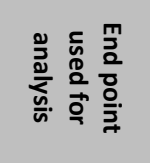 & 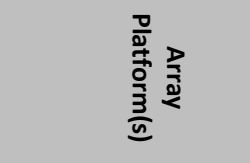 & 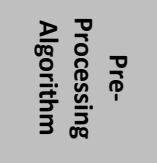 & 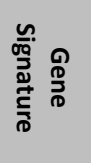 & 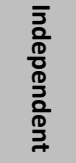 & $\begin{array}{l}\frac{8}{0} \\
\frac{0}{2} \\
\frac{3}{11}\end{array}$ & $\frac{\vec{n}}{\text { d̊ }}$ & 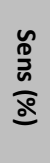 & $\begin{array}{l}\text { ca } \\
0 \\
\frac{0}{0} \\
\text { do }\end{array}$ \\
\hline 品 & $\ddot{o}$ & $\circ$ & $\infty$ & N & 0 & $\circ$ & $\bullet$ & $z$ & $\tilde{\omega}$ & $\dashv$ & $\overline{\bar{z}}$ & Tᄁ & 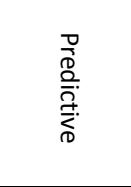 & 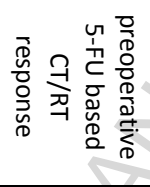 & 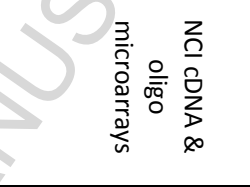 & 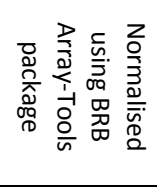 & $\stackrel{G}{\rightarrow}$ & 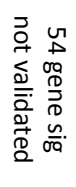 & $v$ & そ & z & z \\
\hline$\stackrel{b}{\omega}$ & $\ddot{o}$ & 0 & 6 & $\stackrel{\sim}{\tilde{H}}$ & $\circ$ & $\circ$ & $\vdash$ & z & $\tilde{\omega}$ & $\dashv$ & $\bar{z}$ & Tᄁ & 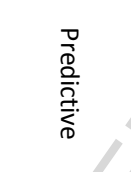 & 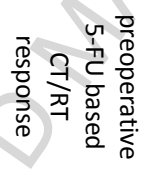 & 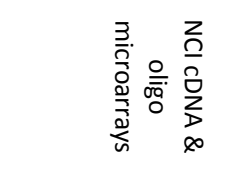 & 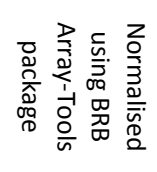 & $\stackrel{G}{a}$ & 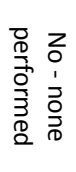 & $\circ$ & z & z & z \\
\hline$\$$ & $\varnothing$ & 0 & 0 & 0 & 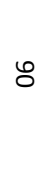 & 0 & 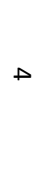 & z & 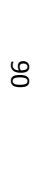 & -1 & ज & Tᄁ & & 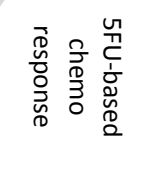 & 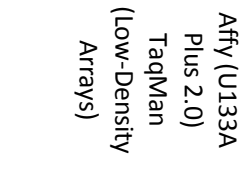 & 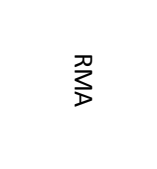 & $v$ & $\begin{array}{ll}\overline{0} & z \\
0 & 0 \\
\frac{1}{7} & 1 \\
0 & 1 \\
3 & 0 \\
0 & 0 \\
0 & 0\end{array}$ & $\circ$ & z & z & z \\
\hline ज & $\stackrel{\sim}{\mapsto}$ & 0 & 0 & 0 & $\tilde{\sim}$ & 0 & $\vdash$ & 同 & $\stackrel{\sim}{\oplus}$ & -1 & $\overline{z_{0}}$ & T & 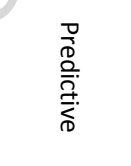 & 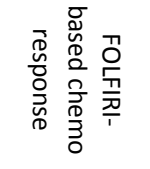 & 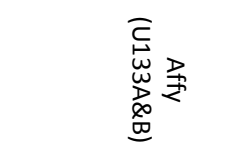 & \begin{tabular}{l}
3 \\
\multirow{2}{*}{} \\
ch \\
0
\end{tabular} & $\stackrel{\vec{A}}{ }$ & $\begin{array}{ll}\overline{0} & z \\
\frac{0}{0} & 0 \\
\frac{7}{7} & 1 \\
\frac{3}{3} & \vdots \\
0 & 0 \\
0 & 0\end{array}$ & 0 & zo & z & z \\
\hline คे & ఎ & 0 & 0 & 0 & 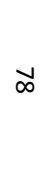 & 0 & $D$ & 同 & w్ర & $\dashv$ & un & ᄁᄁ & 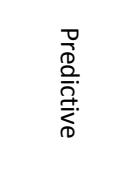 & 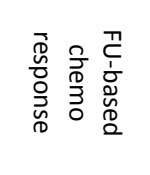 & 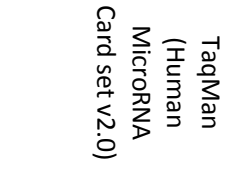 & 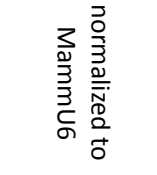 & 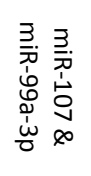 & ఏ & $\underset{\omega}{\omega}$ & $\bar{z}$ & $\bar{z}$ & $\bar{z}$ \\
\hline
\end{tabular}




\section{Table 1C Prognostic Multi-gene Signature Studies}

\begin{tabular}{|c|c|c|c|c|c|c|c|c|c|c|c|c|c|c|c|c|c|c|c|c|c|c|}
\hline \multirow[b]{2}{*}{$\begin{array}{l}\frac{P_{0}}{\mathbb{2}} \\
\frac{\mathbf{z}}{0}\end{array}$} & \multicolumn{9}{|c|}{ Patient Cohort Information } & \multicolumn{3}{|c|}{ Sample Details } & \multicolumn{2}{|c|}{ RNA Profiling Objectives } & \multicolumn{3}{|c|}{ Platform and method to derive multi-gene signature } & \multicolumn{5}{|c|}{ Validation Group Within Original Paper } \\
\hline & 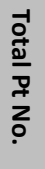 & $\begin{array}{l}\text { 邑 } \\
\text { 品 } \\
⺊ \\
⺊\end{array}$ & 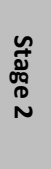 & 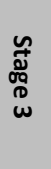 & 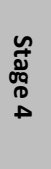 & $\begin{array}{l}\frac{1}{3} \\
\frac{2}{0} \\
\frac{0}{0} \\
\frac{0}{3} \\
0\end{array}$ & $\frac{\underline{\rho}}{\underline{\underline{n}}}$ & 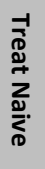 & 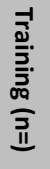 & 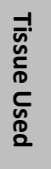 & 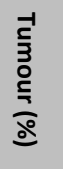 & 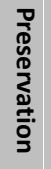 & 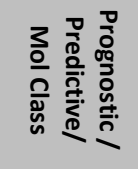 & 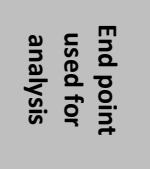 & 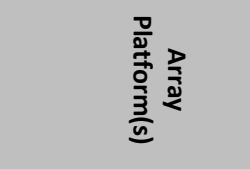 & 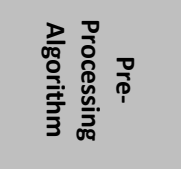 & 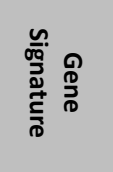 & $\begin{array}{l}\bar{z} \\
\frac{2}{0} \\
\frac{0}{0} \\
0 \\
0 \\
0 \\
0 \\
\frac{0}{7}\end{array}$ & $\begin{array}{l}\frac{\delta}{0} \\
\text { o } \\
\frac{1}{3} \\
\text { II }\end{array}$ & 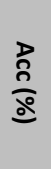 & 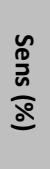 & $\begin{array}{l}\frac{n}{0} \\
\frac{0}{2} \\
\frac{0}{\delta}\end{array}$ \\
\hline$\infty$ & $\begin{array}{l}\infty \\
0 \\
*\end{array}$ & ஜ̆ & $\stackrel{w}{N}$ & $\stackrel{w}{\omega_{0}}$ & $\tilde{v}$ & 0 & 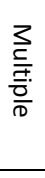 & $\bar{z}_{\mathrm{j}}$ & $\underset{\widetilde{N}}{\tilde{N}}$ & $\begin{array}{l}2 \\
3 \\
+ \\
-1\end{array}$ & $\bar{z}$ & $\bar{z}$ & 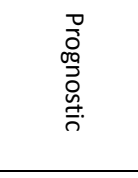 & 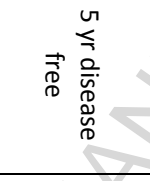 & 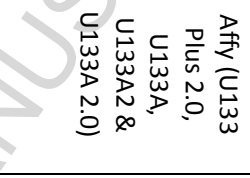 & 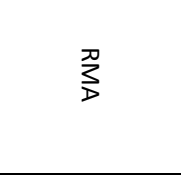 & 胥 & 官 & $\stackrel{\infty}{0}$ & z & z & z \\
\hline$\underset{\perp}{\omega}$ & $\stackrel{\infty}{\infty}$ & $\circ$ & 它 & $\infty$ & $\circ$ & 0 & $\vdash$ & 市 & $\stackrel{\infty}{\infty}$ & $\dashv$ & $\stackrel{v}{\infty}$ & $\pi$ & 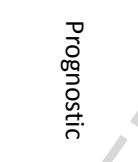 & 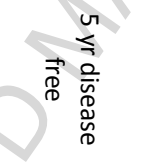 & 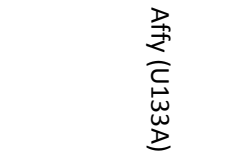 & $\frac{\pi}{3}$ & o & 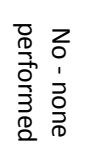 & $\circ$ & z & z & Z \\
\hline$\omega$ & $\underset{\substack{\infty \\
બ}}{\stackrel{\infty}{心}}$ & $\circ$ & 灾 & $\stackrel{\infty}{\not}$ & $\circ$ & 0 & 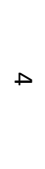 & $\widehat{\infty}$ & 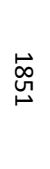 & $\dashv$ & $\bar{z}$ & $\begin{array}{l}\text { 穷 } \\
\text { m }\end{array}$ & 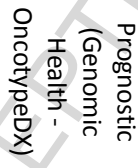 & 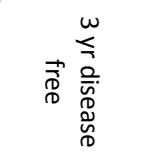 & 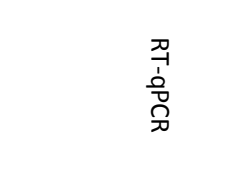 & 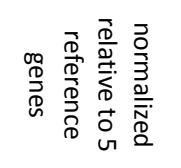 & 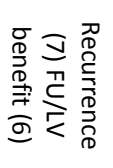 & $\begin{array}{ll}\overline{0} & z \\
\frac{0}{7} & 0 \\
0 & 1 \\
\frac{3}{3} & 1 \\
0 \\
0 \\
0\end{array}$ & $\circ$ & z & z & z \\
\hline $\mathcal{w}_{W}$ & $\underset{w}{\tilde{w}}$ & 0 & $\underset{\omega}{\tilde{\omega}}$ & 0 & 0 & 0 & $\omega$ & 同 & $\underset{\omega}{\tilde{\omega}}$ & $\dashv$ & $\stackrel{\text { IV }}{0}$ & 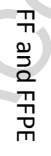 & $\begin{array}{l}\text { ờ } \\
\text { ơ } \\
\text { og } \\
0 \\
\frac{0}{n}\end{array}$ & 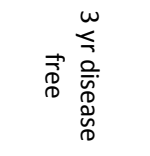 & 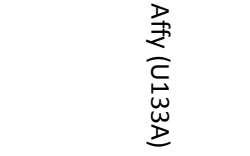 & 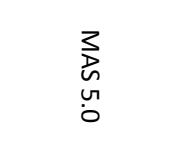 & $v$ & 同 & $\stackrel{!}{\circ}$ & $\bar{z}$ & 요 & $\stackrel{\infty}{\sim}$ \\
\hline w & $\stackrel{\vec{D}}{\vec{b}}$ & $\tilde{\omega}$ & \begin{tabular}{l}
$\omega$ \\
\multirow{0}{0}{}
\end{tabular} & $\overrightarrow{0}$ & $\stackrel{\llcorner}{\sim}$ & $\widetilde{o}$ & $\sum_{i}$ & 同 & $\underset{D}{\mathbb{D}}$ & $\begin{array}{l}2 \\
3 \\
+ \\
-1\end{array}$ & $\bar{z}$ & $\bar{z}$ & 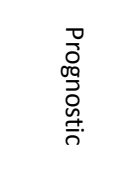 & 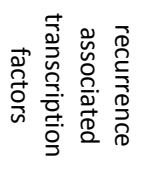 & 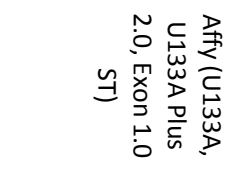 & 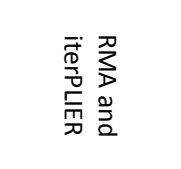 & $\stackrel{\sim}{\mapsto}$ & 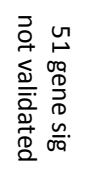 & 0 & z & z & z \\
\hline$\underset{v}{w}$ & $\mathscr{\infty}_{0}$ & $\bar{z}_{0}$ & $\bar{z}$ & $\bar{z}$ & $\bar{z}$ & $\bar{z}_{0}$ & $\sum_{i}$ & $\bar{z}_{0}$ & 홍 & $\dashv$ & $\bar{z}$ & $\bar{z}$ & 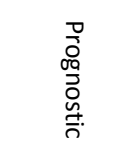 & 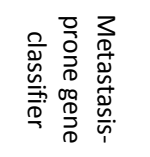 & 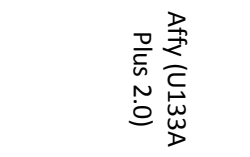 & 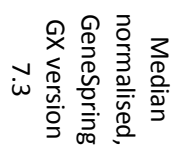 & • & 同 & $\tilde{\sigma}$ & $\stackrel{\infty}{\circ}$ & $\infty_{\infty}^{\infty}$ & œ \\
\hline
\end{tabular}




\section{Table 1C Prognostic Multi-gene Signature Studies (Cont'd)}

\begin{tabular}{|c|c|c|c|c|c|c|c|c|c|c|c|c|c|c|c|c|c|c|c|c|c|c|}
\hline \multirow[b]{2}{*}{$\begin{array}{l}\text { ग्ग } \\
\stackrel{\mathbb{D}}{\mathbf{z}} \\
\stackrel{0}{0}\end{array}$} & \multicolumn{9}{|c|}{ Patient Cohort Information } & \multicolumn{3}{|c|}{ Sample Details } & \multicolumn{2}{|c|}{ RNA Profiling Objectives } & \multicolumn{3}{|c|}{ Platform and method to derive multi-gene signature } & \multicolumn{5}{|c|}{ Validation Group Within Original Paper } \\
\hline & 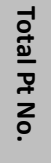 & 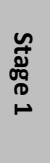 & 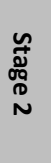 & 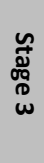 & 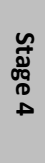 & $\frac{9}{3}$ & 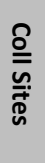 & 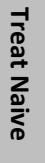 & 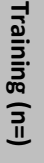 & 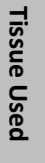 & $\begin{array}{l}\frac{1}{5} \\
\frac{5}{3} \\
\stackrel{0}{9}\end{array}$ & 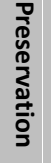 & 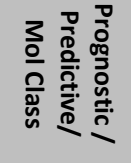 & 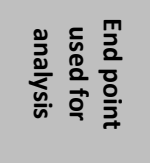 & 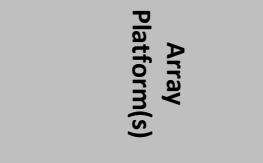 & 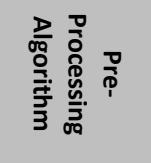 & 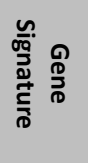 & 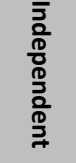 & $\begin{array}{l}\frac{2}{0} \\
\frac{0}{\partial} \\
\frac{1}{3} \\
\text { II }\end{array}$ & $\frac{\vec{n}}{\text { a }}$ & 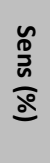 & $\begin{array}{l}\text { con } \\
0 \\
\text { do } \\
\text { do }\end{array}$ \\
\hline$\underset{\infty}{\omega}$ & $\underset{ٍ}{\stackrel{\omega}{\phi}}$ & $\stackrel{U}{\perp}$ & $\stackrel{\widetilde{B}}{\vec{b}}$ & $\stackrel{\vec{\infty}}{\infty}$ & $\infty$ & $\circ$ & 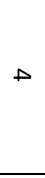 & $\bar{z}$ & $\begin{array}{c}\vec{\infty} \\
\infty\end{array}$ & $\dashv$ & $\bar{z}$ & Tᄁ & 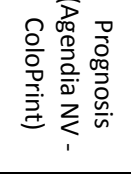 & 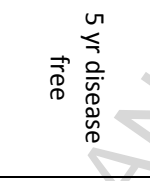 & 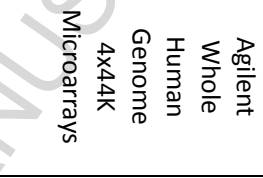 & 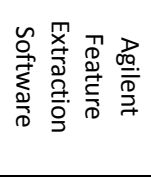 & $\stackrel{b}{\infty}$ & $\widehat{\$}$ & 茴 & z & z & z \\
\hline$\stackrel{\omega}{\omega}$ & G & 0 & $w_{\sim}^{w}$ & 8 & 0 & 0 & $\mapsto$ & 恿 & $\stackrel{\varphi}{u}$ & $\dashv$ & $\infty$ & ᄁᄁ & $\begin{array}{l}\text { 울 } \\
\text { og } \\
\text { वे }\end{array}$ & 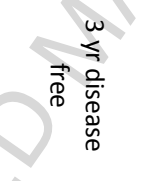 & 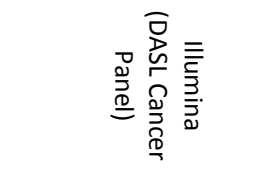 & 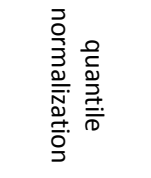 & $\infty$ & 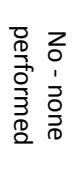 & 0 & z & z & z \\
\hline 古 & $\underset{w}{w}$ & 0 & $\underset{w}{w}$ & 0 & 0 & 0 & $\rightarrow$ & 恿 & v & $\dashv$ & $\infty$ & $\begin{array}{l}\text { 勇 } \\
\text { n }\end{array}$ & & 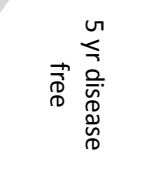 & 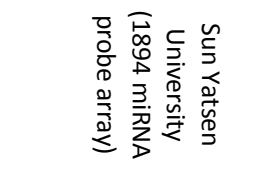 & 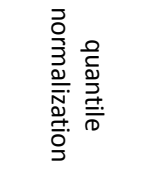 & 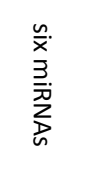 & ঐ & คे & $\bar{z}_{0}$ & $\overline{\bar{z}}$ & $\bar{z}$ \\
\hline$\stackrel{\forall}{\forall}$ & $\stackrel{亡}{\sim}$ & $\vdash$ & $\omega$ & $\omega$ & G & 0 & $\vdash$ & 同 & $\stackrel{\grave{N}}{ }$ & $\dashv$ & $\overline{z_{0}}$ & T & 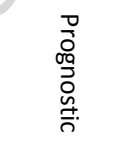 & 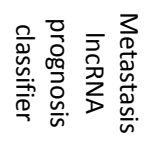 & 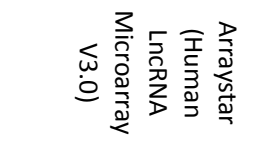 & 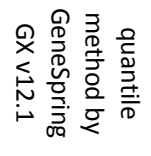 & 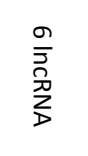 & $\begin{array}{ll}\overline{0} & z \\
\frac{1}{7} & 0 \\
0 & 1 \\
\frac{1}{3} & \vdots \\
0 & 0 \\
0 & 0\end{array}$ & 0 & zo & z & z \\
\hline$\stackrel{\infty}{\infty}$ & $\stackrel{\infty}{N}$ & 0 & $\infty$ & ర) & 0 & 0 & $\begin{array}{l}\frac{3}{\frac{3}{c}} \\
\frac{\overline{\bar{t}}}{\frac{\bar{t}}{0}}\end{array}$ & d & $\begin{array}{l}\infty \\
\infty\end{array}$ & $\dashv$ & $\bar{z}$ & $\begin{array}{l}\frac{7}{n} \\
\text { m }\end{array}$ & 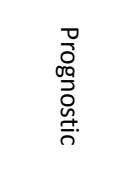 & 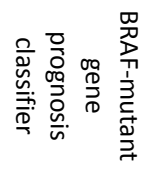 & 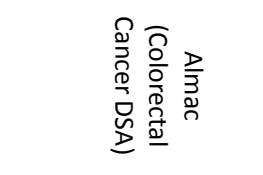 & $\frac{\pi}{\frac{D}{D}}$ & $\stackrel{\circ}{\AA}$ & ఏ & $\underset{\sim}{\stackrel{\leftrightarrow}{\sim}}$ & z & டூ & $\stackrel{\infty}{\circ}$ \\
\hline
\end{tabular}


Table 1. Summary of CRC GEP studies. Variables which can impact on the success of gene signature development were detailed and for the purposes of the table, grouped into 5 overarching categories: patient cohort information; sample details; RNA profiling objectives; array platform and methodology used to derive multi-gene signatures; and validation group within original paper. Data were recorded for a select number of studies relating to (A) molecular taxonomy/classification studies (B) predictive signatures and (C) prognostic signatures. Abbreviations used in table are as follows; specimen collections sites (coll sites), treatment naïve (treat naïve), molecular classification (mol class), accuracy (acc), sensitivity (sens), specificity (spec), non-malignant tissue (NM), malignant tissue (T), robust multiarray analysis (RMA), frozen RMA (fRMA), Affymetrix (affy) and information not provided (INP). The full list of publications reviewed for this study can be found in Supplementary table 1 .

\section{Variable 1 - Quality and quantity of samples}

Prior to commencing array-based or RNA-seq studies, it is important to determine if sufficient samples (with adequate malignant tissue percentages) are available for analysis. As discussed earlier, gene lists developed for classification, prediction or prognosis or treatment response exhibit little commonality between studies and often are heavily dependent on the training group selected from the overall cohort. From reviewing supplementary table 1 there is a marked difference in the number of genes included within a gene signature with some signatures having less than 10 genes whereas one publication used 1042 genes to perform molecular classification. Some papers suggest to have a small number of genes within a gene signature to enable the test to be transferred to other platforms such as quantitative $\mathrm{PCR}^{27}$. One group has used a mathematical model called the "probably approximately correct" learning technique to predict the number of patients required within a randomly assigned 
training cohort to enable a $50 \%$ overlap between 70 -gene signatures generated from the randomly assigned training cohorts ${ }^{27}$. This study indicated that 2000-3000 samples are required in order to generate a robust 70 -gene signature, yet of the 57 publications we identified from PubMed searches, only one study had over 2000 samples in the training cohort $^{27}$. Two studies used only cell lines and 33 studies had fewer than 100 patients in the training cohort, providing a potential explanation as to why many signatures fail to demonstrate clinical value during validation on independent cohorts.

Transcriptomic-based biomarker development must conform to appropriate ethical approval, attention to clinical and pathological characteristics, inclusion/exclusion study criteria and consultation with clinicians and statisticians. We noted a large disparity in tumour staging and tumour content, preservation method, treatment status and metastatic status in studies reviewed in Table 1. In addition, some studies were performed on a single site whereas others were across multiple sites, with little evidence of standard operating procedures (SOPs) being applied across study centres. Introduction of sampling bias must be considered as tissue blocks selected based on availability of tissue may be biased towards more bulky tumour subtypes. Defining sample type, method of preservation/storage, transportation, tissue area to macrodissect and volume of malignant tissue required by the transcriptomic facility is essential in order to account for all these variables at the analytic stage ${ }^{84}$. Determining the projected attrition rates throughout the analytical pipeline, by firstly performing a pilot study, will allow an informed assessment of minimum tissue requirements and a better estimation of whether sufficient samples exist ${ }^{85}$. Before study commencement, SOPs should be developed to describe tissue procurement, processing, storage, purification and quantitation of nucleic acids and microarray protocols employed. 


\section{Variable 2 - Tissue Resection}

During surgical resection, the type of drugs employed (including anaesthetics) and the duration of exposure in patients, can have substantial effects on subsequent RNA profiles ${ }^{86}$. In surgery, reducing warm (disruption of blood supply while removing the tissue) and cold (time between tumour removal and formalin fixation) ischemia times can improve RNA integrity. Some studies indicate CRC tissue shows little variation in GEP with changes in warm or cold ischaemia times whereas other studies have demonstrated a tissue-specific degradation of RNA with extended ischaemia times ${ }^{87-90}$. Regulator of G-protein signalling 1 (RGS1) and eukaryotic translation elongation factor 1 alpha 1 (EEF1A1) have been identified as potential markers of primary colorectal carcinoma tissue quality and post-operative tissue quality respectively ${ }^{91}$. Therefore warm/cold ischaemia duration, type of drugs administered during surgery, along with type and location of resected tissue should be recorded as potential confounding variables for array-based or RNA-seq studies of tissue derived from different organs.

\section{Variable 3 - Formalin fixation and paraffin embedding effect on mRNA}

AJCC staging requires histopathological examination of resected tumours. Only six of the 57 $(11 \%)$ studies that we reviewed were exclusively performed on FFPE tissue, while two studies (4\%) used both FF and FFPE material from different cohorts, with the remainder of studies being FF or the information was not provided. A FF tissue GEP signature may also require validation with FFPE material to ensure probesets are not adversely affected by formalin fixation ${ }^{17}$. Formalin fixation alternatives do exist (Z7, RCL2®, PAXgene ${ }^{\circledR}$, Allprotect ${ }^{\circledR}$ and RNAlater $\left.{ }^{\circledR}\right)$, although formalin fixation is considered the standard processing method in clinical practice, and the reference approach for routine diagnostics validations 92,93 . 
Increasing tumour size leads to increased time for the formalin to penetrate the inner core, resulting in potential tissue autolysis ${ }^{94}$. Therefore, potential differences in gene expression profiles observed between small biopsy material and large resection material may simply arise from differences in formalin penetration and fixation times that are dependent on tumour size. FFPE recommendations for nucleic acid studies include ensuring pre-fixation time is minimised, use of cold $\left(4^{\circ} \mathrm{C}\right) 10 \%$ neutral buffered formalin and limiting the duration of fixation (3 to 6 hours) ${ }^{94}$. RNA integrity from FFPE tissue is poorer in comparison to snap frozen, although some studies indicate that having a quick, uniform FFPE method can lead to comparable results ${ }^{95}$. However, working with FF samples also has its difficulties in routine practice, and a degree of attrition is equally expected with this type of sample.

While SOPs should be implemented in transcriptomic studies, investigators need to be wary, particularly in retrospective studies where clinical material is being received from multiple sites, that processes such as tissue fixation are often not standardised and can lead to variation in resulting molecular studies ${ }^{96,97}$. Therefore, variations in tumour blocks from different hospital sites, surgical resection of the tumour, pre-fixation times and methods should be recorded, and the effects of these variables on subsequent array-based or RNA-seq derived molecular signatures examined.

\section{Variable 4 - FFPE Tumour Block Storage}

Following FFPE processing, initial guidelines recommended blocks are stored without protection from light or air, provided the uppermost section is discarded for RNA preparations and extractions are performed within a year ${ }^{98}$. Improvements in RNA extraction, in vitro transcription (IVT) and microarray platforms have led an increasing 
number of studies utilising FFPE blocks which have been stored for longer than a year, with a successful RNA profiling outcome ${ }^{99}$. The S:CORT programme has performed RNA profiling on FFPE tumour blocks from randomised control trials which have been stored at room temperature for up to 21 years. Figure 2 illustrates that we did not identify a correlation between tumour block age and the following parameters; total RNA 260/280 and 260/230 ratios, total RNA yield, cRNA yield, QC metrics such as $3^{\prime} / 5^{\prime}$ ratio of actin or the overall success rate for transcriptional profiling of the sample using the FFPE-optimised Almac Xcel Array. On the Xcel array, probes which have been shown to be stable or having comparable longitudinal stability during formalin fixation or subsequent storage were included. For arrays containing probes which have not been optimised for FFPE material, we recommend recording FFPE tumour block storage duration and using material for RNA extraction within 2 weeks of sectioning from the block to ensure storage-dependent RNA degradation does not further compromise any array-based or RNA-seq study derived signature.

A

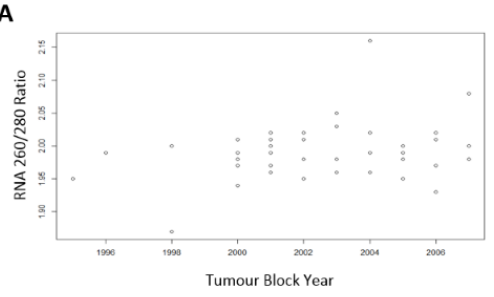

D

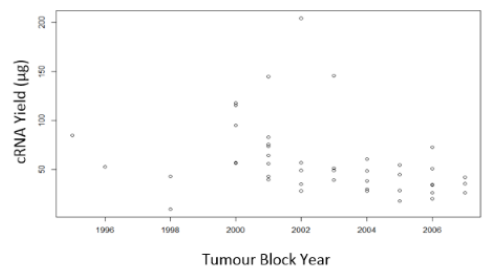

B

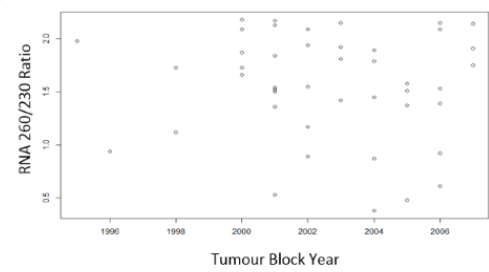

E

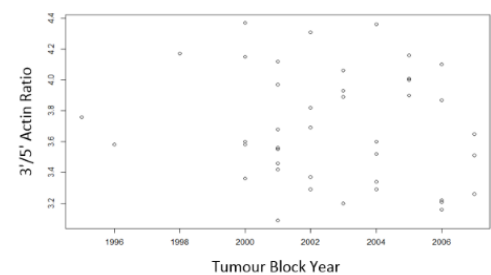

C

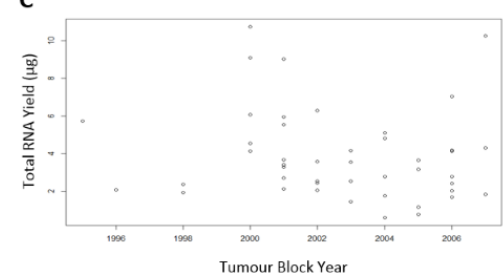

F

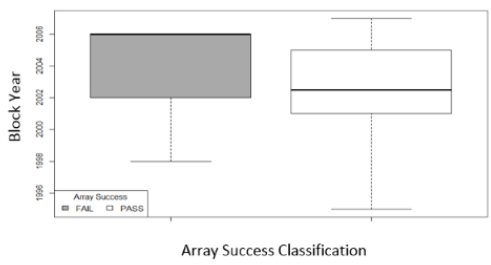

Figure 2. Assessment of the effect of tumour block age on RNA and cRNA parameters and success in generating a CEL file. RNA extraction was performed using the Roche High Pure RNA Paraffin Kit and the resulting 260/280, 260/230 and total RNA yield for each sample was recorded. During the Affymetrix 3' IVT PLUS Reagent Kit stage, 260/280, 260/230 and total yields for cRNA and double stranded cDNA were recorded. Following 
hybridisation, washing and scanning, the CEL files were normalised using Robust Multiarray Average (RMA) and QC metrics, including actin $3^{\prime} / 5^{\prime}$ ratio, were examined using Affymetrix Expression Console Software. Dot plots examining the relationship between tumour block year and total RNA 260/280 (A), total RNA 260/230 (B), total RNA yield (C), cRNA yield (D), actin 3'/5' ratio (E) and whether the sample was subsequently hybridised to an $\operatorname{array}(\mathbf{F})$.

\section{Variable 5 - Tissue macrodissection}

Prior to commencing the study, the minimum quantity of RNA that can be analysed using the laboratory's standard RNA profiling protocol and selected GEP platform, without confounding the resulting RNA profile, needs to be determined. This will enable investigators to estimate tissue quantity requirements and therefore determine how many sections, of a specified thickness, need to be prepared from each tumour block. In our current S:CORT transcriptome profiling study, when using biopsy material, the annotated tumour region can be very small (surface area $<20 \mathrm{~mm}^{2}$ per section) and therefore require a larger number of sections to be prepared in comparison to the number of sections prepared from resection blocks. This is particularly relevant if the study involves limited biopsy material, or if a multi-omics approach is required from the same tissue block.

An additional section is prepared for $\mathrm{H} \& \mathrm{E}$ staining to enable a pathologist to mark areas of tumour presence and assess tumour content. Tumour cell isolation from FFPE sections requires macrodissection of pathologist-annotated tumour regions, which is prone to individual subjectivity ${ }^{100}$. As tumours are not uniform in shape throughout the tissue block, many investigators prepare consecutive sections, placed onto numbered slides corresponding with the order that they were sectioned in, with the top, intermediate and bottom sections 
being used for H\&E staining ${ }^{101}$. Table 1 highlights that the tumour content percentage acceptable for the published studies is not reported in over half the studies, or large variations in acceptable tumour content (mean 68\% with a range of 30-90\%) are reported. A high degree of variation exists between different pathologists' assessment of tumour content in defined samples, which could ultimately diminish the prognostic/predictive power of any resultant GEP signature ${ }^{102}$. In collaboration with an industrial partner, our laboratory has developed a digital pathology platform for automated tumour annotation and percentage tumour nuclei measurement, which correlates well with benchmark tumour cell counts ${ }^{100}$.

Studies assessing the impact of lower tumour content (cellularity) highlight that pathological factors such as the presence of invasive cells and budding at the tumour margin can impact on the expression profile and be predictive of survival ${ }^{103}$. The tumour content threshold and the number and type of cells present in the non-tumour areas can have a major impact on the expression level of specific genes. Inaccurate assessment of tumour content may impact on GEP-based clinical diagnostics and GEP-based methods to evaluate tumour content may need to be developed to complement the pathologist assessment ${ }^{104}$. In CRC, two of the four consensus CMS groups are based on relative changes in the stromal content, namely increasing fibroblasts (CMS4) or immune cell infiltration (CMS1) in the tumour microenvironment, with the type and location of immune cells having a well-defined impact on clinical outcome ${ }^{105}$. Microarray profiling of multiple regions within individual CRC FFPE samples by our group has highlighted that the region of tumour used for GEP can confound patient CMS classification, further confirming how CRC classification signatures are fundamentally affected by intratumoural stromal heterogeneity as well as the underlying tumour biology ${ }^{84}$. A further complication is the differences in the GEP from primary tumour and metastatic sites, arising from the differing tumour microenvironment, underlying 
biological factors such as clonality in the primary tumour, or metastatic cells having undergone an epithelial mesenchymal transition ${ }^{84}$. Creating an electronic repository of the pathologist annotated H\&E images will enable researchers to review images to validate arraybased or RNA-seq derived multi-gene signatures, which may be indicative of immune or fibroblast cell infiltration.

\section{Variable 6 - RNA Extraction}

Developments in commercial total RNA extraction kits have led to more FFPE tumours becoming suitable for analysis. Although there are protocol variations between kits, they typically involve tissue dewaxing by a solvent such as xylene and sequential ethanol hydration steps. Recent non-toxic approaches have been reported, such as elevated heat, sonication or proprietary buffers to remove the paraffin. This is followed by macrodissection prior to enzymatic proteolysis of the tissue by a proteinase $\mathrm{K}$ incubation. It is reported that proteinase $\mathrm{K}$ can reverse mono-methyl nucleotide modifications and break the RNA-protein cross-links formed during formalin fixation ${ }^{95,106}$. A DNase step is often included to remove genomic DNA contamination from downstream applications, while some report additional steps to reverse RNA cross-linking which occurs during formalin fixation. RNA purification is commonly performed using spin column-based technology, utilising glass fibres or suitable matrices. Contaminants and/or carry-over of nucleic acid based carriers during RNA purification can inhibit downstream enzymatic applications and therefore compatibility with downstream array based technologies needs to be assessed.

Recent research also suggests other RNA populations, such as microRNA (miRNA), may be

more stable in samples with heavily degraded mRNA, particularly from FFPE tissue ${ }^{107}$. Investigators may therefore require either array-based or RNA-seq platforms to quantitate 
levels of small RNA populations such as miRNA. For both array-based and RNA-seq studies, it is currently not possible to measure mRNA and miRNA transcripts simultaneously. RNA-Seq of mRNA and miRNA require different chemistries used for ligation of RNA-Seq adaptors during library preparation protocols and array based platforms use different chemistries for ligation of the biotin signalling molecule to miRNA or templates derived from mRNA. Therefore, separate protocols for analysis are required and the financial feasibility of running both mRNA and miRNA expression studies in tandem needs to be considered. For investigators looking to quantitate miRNA using either an array-based or RNA-seq approach, ensuring the RNA extraction protocol will retain small RNAs ( $<200$ nucleotides) is essential, as it has been reported that commercial RNA extraction kits exhibit variable success in retaining small non-coding RNAs ${ }^{108}$.

\section{Variable 7 - Quantitation and Assessment of RNA Quality}

The FFPE process leads to extensive RNA fragmentation and cross-linking, impacting on RNA profiling success. A combination of RNA quantitation and integrity/quality assessments are warranted. There are a number of spectrophotometric (Nanodrop) and fluorescence based (Qubit and Quant-iT) methods for RNA quantitation. Spectrophotometric methods require little preparation time and function by measuring absorbance at $260 \mathrm{~nm}$ to determine nucleic acid quantity, with measurements at $230 \mathrm{~nm}$ and $280 \mathrm{~nm}$ indicative of solvent and protein contamination respectively ${ }^{109}$. Unfortunately, spectrophotometric methods can report artificially high concentrations, due to simultaneous measurement of both RNA and DNA readings within the sample. Fluorescence-based methods have suitable levels of sensitivity for RNA, although the dynamic measurement range is smaller than for spectrophotometric methods, with a frequent requirement for sample dilution, resulting in longer assay times ${ }^{110}$. Due to FFPE-dependent fragmentation of RNA, a separate method to examine RNA integrity 
is often reported, using the Agilent 2100 Bioanalyser along with a proprietary algorithm to establish a RNA integrity number (RIN). There is no consensus threshold RIN value for downstream applications and we noted large variations in this threshold value between studies $40,53,54,63,111$. From our microarray experience, we have generated RNA profiling data, deemed successful by assessment of QC metrics such as 3'-5' ratio of housekeeping genes, from samples with RIN values as low as 1.3. FFPE also results in chemical modification of nucleic acids and crosslinking with proteins, which would not be detected by the Agilent 2100 Bioanalyser ${ }^{98}$. Performing a PCR-based screen to assess RNA sample quality prior to proceeding to analysis on a comparatively expensive array is recommended ${ }^{112}$.

\section{Variable 8 - RNA Profiling Platform}

The Biorepositories and Biospecimen Research Branch (BBRB) of the Cancer Diagnosis Program (CDP) at the National Cancer Institute advises the use of validated assays, standardized training of technical staff in the performance of the assay, lot uniformity of reagents, inclusion of appropriate type and number of quality control samples, randomisation when possible and standardised methods for documenting and interpreting testing results ${ }^{113}$. If a GEP signature is to be developed that can harness the power of large archived clinically annotated cohorts of FFPE tumour blocks, then inclusion of samples with moderate to severe RNA degradation will need to be accommodated, impacting on array platform selection. Studies have demonstrated specific mRNA transcripts are more susceptible to degradation, due to FFPE block processing or storage ${ }^{61,114}$. The $5^{\prime}$ end of the mRNA transcript is more sensitive to FFPE-dependent RNA degradation, resulting in development of arrays with probe designs focused on the extreme 3' of mRNA (Affymetrix Human X3P Array \& Almac Xcel Array) as these regions are more stable in FFPE tissue. The use of IVT reagents specifically designed for FFPE-extracted RNA which include both oligo-dT primers (bind to polyA tail 
and specific for mRNA) and random hexamers (anneal throughout the target molecule) for first strand cDNA synthesis. This enables amplification at the 3 ' end and throughout the whole transcript, which is more applicable to partially degraded mRNA. In GEP studies, recording the type of IVT, labelling and hybridisation kits and associated lot numbers, performance at intermediate steps (such as cRNA and CDNA quantity), dates of IVT/hybridisation/scanning, hybridisation time, chip lot number and operator ID is advised (Table 2).

\begin{tabular}{|c|c|}
\hline Category & Recorded Variables \\
\hline \multirow{6}{*}{ Tumour Block } & Hospital Site \\
\hline & Anaesthetic Used \\
\hline & Warm \& Cold Ischaemia Times \\
\hline & Sample Type (Biopsy / Resection) \\
\hline & Tumour Block Age \\
\hline & Tumour Block ID \\
\hline \multirow{5}{*}{ Macrodissection } & Macrodissection Date \\
\hline & Pathologist Responsible for Annotation \\
\hline & Electronic repository location of annotated images \\
\hline & Section Thickness $(\mu \mathrm{M})$ \\
\hline & No. of Sections Macrodissected \\
\hline \multirow{3}{*}{ RNA Extraction } & $\begin{array}{l}\text { RNA Extraction Kit Manufacturer } \\
\text { RNA Extraction Kit Catalogue No. }\end{array}$ \\
\hline & RNA Extraction Kit Lot No. \\
\hline & RNA Extraction Date \\
\hline \multirow{7}{*}{ RNA Quantitation / Integrity } & RNA Quantitation Method \\
\hline & RNA Concentration $(\mathrm{ng} / \mu \mathrm{l})$ \\
\hline & RNA 260/280 ratio \\
\hline & RNA 260/230 ratio \\
\hline & RNA Quantitation Date \\
\hline & RNA Integrity Measurement Date \\
\hline & RIN Value \\
\hline \multirow{4}{*}{ Microarray Dates } & IVT Date \\
\hline & Technician Performing IVT Process \\
\hline & Hybridisation Date \\
\hline & Scanning Date \\
\hline \multirow{6}{*}{ IVT Information } & IVT Starting Amount of RNA (ng) \\
\hline & cRNA Concentration $(\mathrm{ng} / \mu \mathrm{l})$ \\
\hline & cRNA $260 / 280$ ratio \\
\hline & cRNA 260/230 ratio \\
\hline & ds-cDNA Concentration $(\mathrm{ng} / \mu \mathrm{l})$ \\
\hline & ds-cDNA $260 / 280$ ratio \\
\hline
\end{tabular}




\begin{tabular}{|l|l|}
\hline \multirow{2}{*}{ Hybridisation } & ds-cDNA 260/230 ratio \\
\hline \multirow{5}{*}{ Scanning Information } & Array Type \\
\hline & Array Catalogue No. \\
\hline & Array Lot No. \\
\hline & Hybridisation Start Time \\
\hline & Hybridisation Finish Time \\
\hline & Hybridisation Total Time \\
\hline & DAT Image \\
\hline & Electronic Location of CEL File \\
\hline
\end{tabular}

Table 2. Pre-analytical and analytical variables. Suggested pre-analytical variables which can be recorded to help determine/minimise technical variable-dependent artefacts occurring during gene signature development. Analytical variables can be detailed to monitor performance of samples through RNA profiling and to enable development of exclusion/inclusion sample quality criteria.

\section{Variable 9 - Quality Control (QC) Pipeline}

Development of a QC pipeline for array-based or RNA-seq platforms, integrated into the SOP, enables monitoring of the successful preparation of a sample through pre-analytical and analytical steps. From a pre-analytical perspective, samples are blinded by assigning unique codes so operators are unaware of related clinical information. In large microarray studies, controlling batch effects is required to minimise artefacts occurring in transcriptomic signatures due to non-biological sample variation. Batch effects result from changes in room temperature, ozone level, sample degradation over time, changes in reagent lot numbers, different technical staff or equipment performance variation ${ }^{115}$. The Cancer Genome Atlas (TCGA) data has been generated from different platforms, laboratories and over different time periods, resulting in batch effects which has been problematic ${ }^{115,116}$. This led to TCGA developing statistical batch effect identification tools, enabling implementation of additional statistics to account for batch effects in downstream analyses ${ }^{115}$. 
From an analytical perspective, a positive control is run alongside all batches, which can be sourced from the manufacturer or a third party. Alternatively, other investigators replicate the sample type being analysed in the study, which for the purposes of this review would be a FFPE treated CRC cell line. Employing a quality control chart such as a Levey-Jennings plot, to monitor the GEP performance of the RNA control over a period of time, highlights sample runs outside an operator defined number of standard deviations. Controls for the IVT and hybridisation stages are also advised to be included and more detail has been provided on the online supplementary files ${ }^{117-122}$.

Examination of post-analytical variables commences with the initial QC following scanning by visually image inspection to identify abnormalities including dimness, uneven signal intensity, haze bands, crop circles, scratches, bubbles and areas of poor hybridisation (Figure 3). 

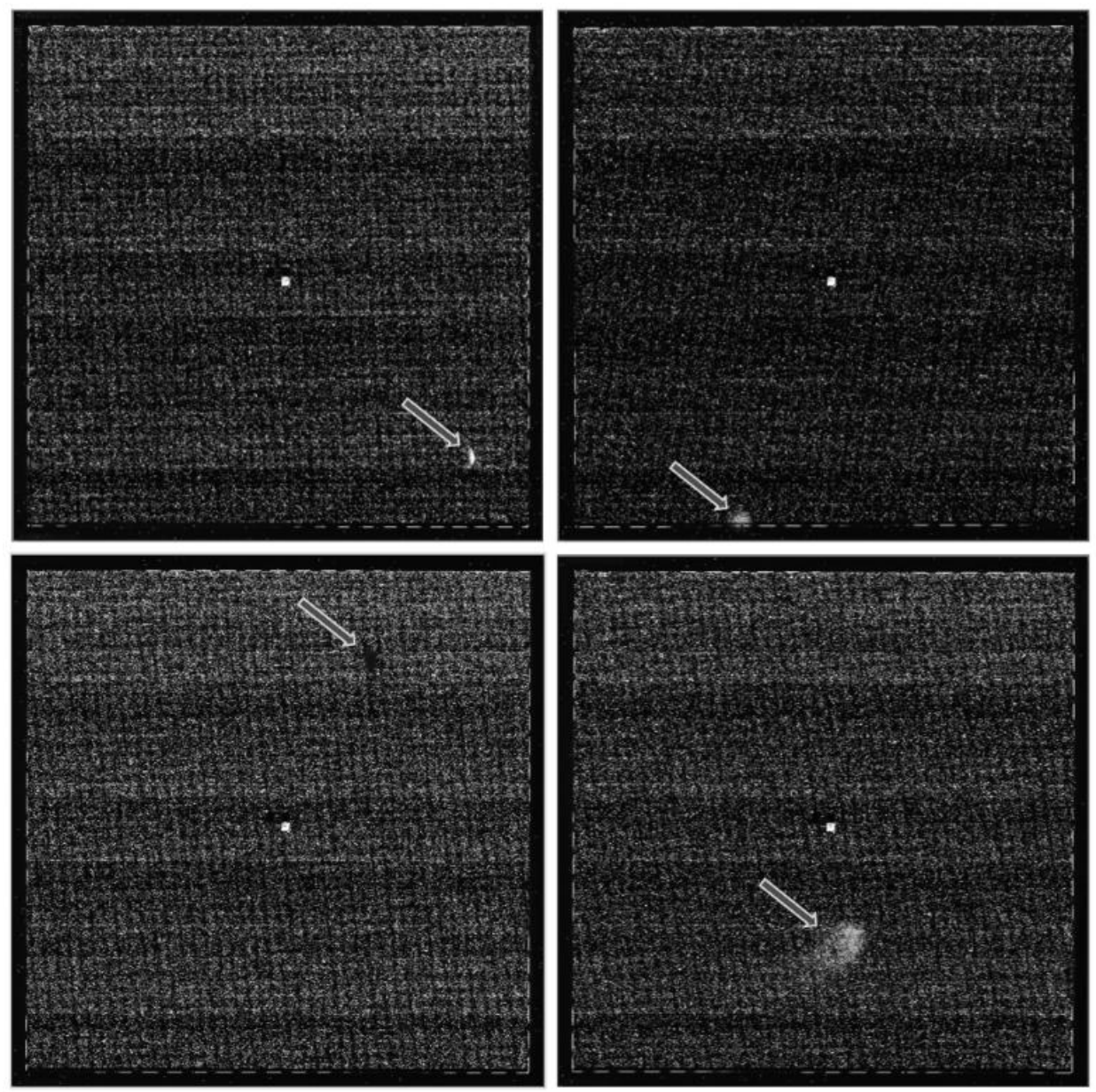

Figure 3: Assessment of the scanned Affymetrix Genechip array image for

abnormalities. The images were accessed using the Affymetrix AGCC viewer function which are generated from the DAT file, which contains the pixel intensity values measured during scanning. Red arrows indicate areas of abnormality in the images from four CRC samples hybridised to the Almac Xcel array.

It is important that dry lab analytical processing methods have a similar degree of validation, maintenance, document-control and quality assurance as wet lab procedures ${ }^{123}$. Subsequently, data pre-processing is performed using commercial or open source analytical platforms to perform a three step process comprising (a) background adjustment, (b) normalisation and (c) summarisation. To ensure hybridisation differences between arrays are accounted for, measurements from different arrays undergo normalisation to ensure array 
results are comparable ${ }^{124}$. Normalisation algorithms (MAS5, RMA, GCRMA etc) are different normalisation methods which will lead to different final expression values and therefore the normalisation method should be reported ${ }^{124-128}$. For IVT QC, an assessment takes three different approaches (i) positive control performance assessment, (ii) assessing endogenous RNA integrity through RNA degradation plots or examination of 3' to 5' ratios of housekeeping genes such as GAPDH, (iii) assessing the signal intensity from spike-in polyA or ERCC controls. For hybridisation QC, assessing the signal intensity from the four hybridisation control transcripts ensures consistent hybridisation within batches. Additional QC metrics related to signal distribution and array comparison can be performed to ensure all samples in a batch are within established thresholds. In the absence of an external quality assessment/proficiency scheme we, similar to other RNA profiling studies, have examined concordance in gene expression profiles from 42 samples measured both at our department and an independent transcriptome profiling centre (Figure 4) ${ }^{129,130}$. 


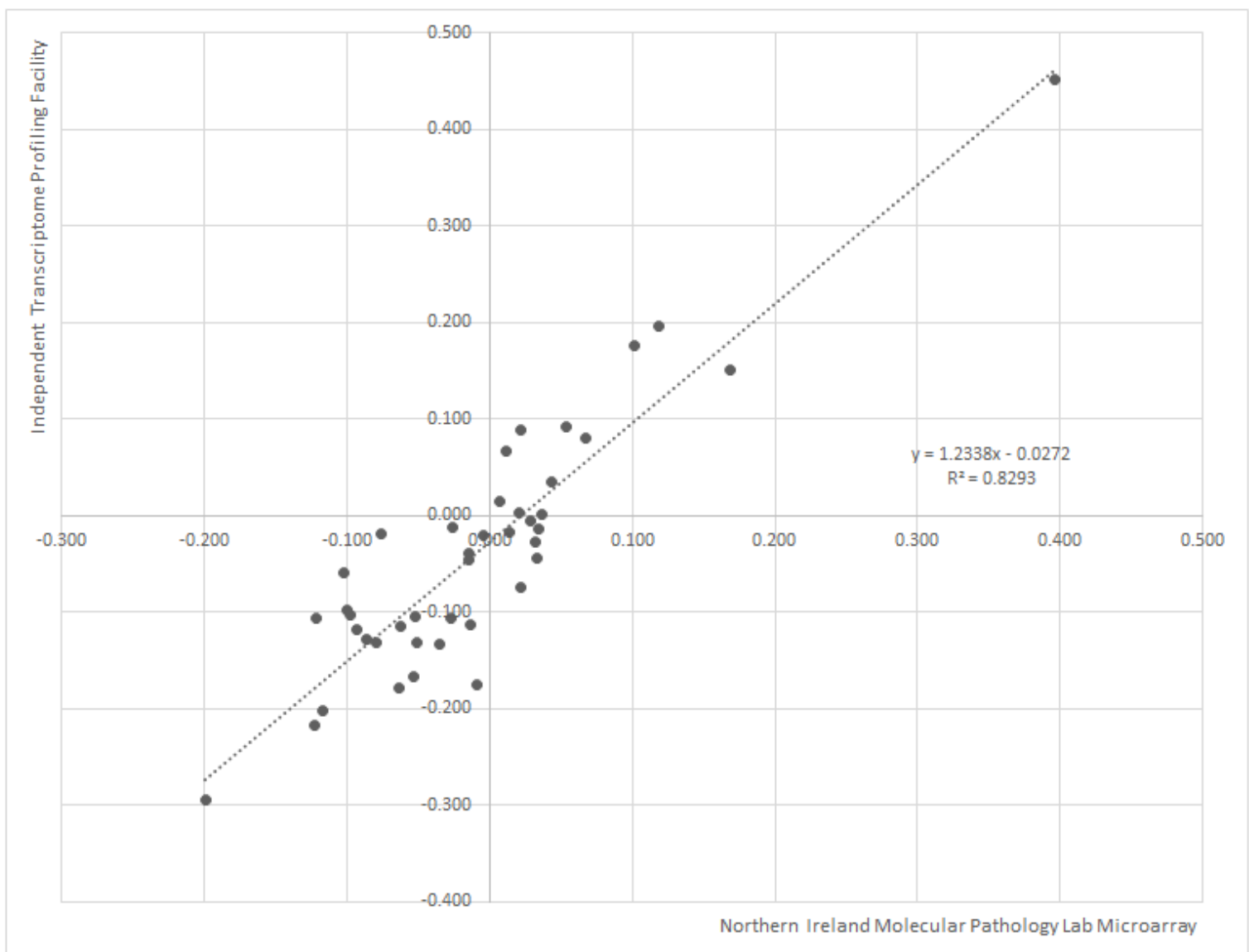

Figure 4: Concordance assessed using a published gene expression score, between RNA profiles measured at our laboratory and an independent transcriptome profiling facility using FFPE CRC samples. Consecutive sections from the same 42 FFPE tumour blocks were sent to two different RNA profiling facilities. RNA extraction was performed using the Roche High Pure RNA Paraffin Kit, 3' IVT target preparation and labelling was performed using the Affymetrix 3' IVT PLUS Reagent Kit and GeneChip Hybridization, Wash, and Stain Kit (Site 1) or the NuGEN Ovation FFPE WTA System and Encore Biotin Module (Site2). Hybridisation, scanning and CEL file generation was performed in an identical manner using the Almac Xcel array.

A QC metrics report, including thresholds, accompanied by justification for inclusion or exclusion of samples from analysis should be included in publications [Table 3]. Furthermore, in the development of RNA profiling-based clinical diagnostic biomarker(s), 
QC data may provide invaluable information for the creation of inclusion/exclusion sample quality criteria. Tables 2 and 3 detail pre-analytical and analytical variables we record within the S:CORT programme to enable us to identify factors which may impact on GEP success [Table 2].

\begin{tabular}{|c|c|}
\hline Category & Recorded Variables \\
\hline \multirow[t]{2}{*}{ Data Image File } & Bubbles, Scratches, Uneven brightness \\
\hline & Intensity of Image Brightness \\
\hline \multirow{7}{*}{ QC Array Metrics (IVT Process) } & Positive Control (Commercial RNA or FFPE) Cell \\
\hline & 3'/5' for GAPDH and Actin \\
\hline & RNA Degradation Plot \\
\hline & lys Average Signal Intensity \\
\hline & phe Average Signal Intensity \\
\hline & tyr Average Signal Intensity \\
\hline & dap Average Signal Intensity \\
\hline \multirow{4}{*}{ QC Array Metrics (Hybridisation) } & bioB Average Signal Intensity \\
\hline & bioC Average Signal Intensity \\
\hline & bioD Average Signal Intensity \\
\hline & Cre Average Signal Intensity \\
\hline \multirow{4}{*}{ QC Signal Quality/Distribution } & Scale Factor \\
\hline & Signal Histogram \\
\hline & Feature Intensity Box Plot \\
\hline & Signal Box Plot \\
\hline \multirow{3}{*}{ QC Array Comparison } & PCA Analysis \\
\hline & Pearsons or Spearmans Correlation Plots \\
\hline & Hierarchical Clustering \\
\hline
\end{tabular}

Table 3. Quality control metrics. A list of quality control metrics which can be examined to ensure robust high quality data is included during downstream analysis. Lys, phe, tyr and dap are bacterially expressed RNA transcripts which have been modified to include a poly-A tail. Four transcripts encoded from E.coli genes bioB, bioC, bioD and the bacteriophage P1 recombinase gene, Cre, are utilised for hybridisation controls. PCA, principal component analysis. 
Prior to commercialisation of any multi-gene prognostic test, the gene expression signature will need to be validated on independent clinical cohorts. As shown in Tables 1A-C, and also in the full table accessible through the online supplementary data, we detail the limited number of published studies which perform an independent validation. Aside from validation of the relevance of the genes involved in the biomarker signature and ensuring the sensitivity and specificity of the test, additional work is necessary to validate the assay performance prior to commercialisation. Intra- and inter-assay precision, accuracy, linearity and sensitivity are required to be investigated for the specific assay. While sensitivity assessment of an immunoassay for a protein or hormone is relatively simple to conduct, RNA profiling from a tissue biopsy has additional complications such as ascertaining the tissue limitations that may limit the generation of sufficient RNA. Factors such as macrodissected area of the biopsy FFPE section, tumour cellularity and the impact of infiltration from fibroblasts and immune cells will also impact on gene express this threshold will be of use.

\section{Commercialisation of transcriptomic based biomarkers}

Several commercial multi-gene CRC prognostic tests have been developed from transcriptomic studies and the commercial product names are listed in the Prognostic / Predictive column in supplementary table 1. Commercial and research-based GEP signatures often result in a gene score, which is a continuous variable, with an assigned threshold to dichotomise samples ${ }^{131-134}$. When technical variability, as detailed in the nine variables above, occurs during transcriptomic analysis, this can lead to misclassification of GEPderived risk scores close to the assigned threshold ${ }^{128,130}$. Manufacturers suggest requesting an additional sample from a different area of the tumour/body to repeat RNA profiling or to consider the GEP risk score alongside clinical parameters of prognostic/predictive value before reaching a final decision that may impact on therapy ${ }^{62,128}$. Given the complexity and 
inherent variability of the technology, GEP companies adopt a centralised lab approach and perform the test as a laboratory developed test (LDT) ${ }^{135}$. The centralised lab operating the LDT is subject to Clinical Laboratory Improvement Amendments (CLIA) laboratory testing processes (the ability to perform laboratory testing in an accurate and reliable manner). The LDT currently falls outside of FDA scrutiny for both the analytical (specificity, sensitivity, accuracy and precision) and clinical validity of in vitro diagnostic tests ${ }^{136}$. Therefore, LDT manufacturers can avoid clinical validation costs and are not subject to the same stringent pre-market review or post market surveillance as an FDA approved in vitro diagnostic would be required to undergo ${ }^{137}$.

\section{$\underline{\text { Conclusion }}$}

In precision medicine, there are continued discussions on what are the most optimal actionable biomarkers (i.e. biomarkers that will indicate particular therapeutic intervention). DNA-based biomarker assays are robust, but the number of actionable mutations is modest, resulting in clinical benefit for a limited number of patients. Protein expression by immunohistochemistry is widely available and relatively affordable, but there are limited informative markers and QA/QC technique concerns ${ }^{138}$. Delineating the transcriptional landscape has the potential to identify robust signatures that may inform stratification of patients for different treatment options. Future molecular transcriptomic approaches, undertaken by international consortiums with large cohort sizes, will require high-throughput standardised methodologies.

From published CRC GEP studies, we report pre-analytical, analytical and post analytical variables within a GEP study, where adoption of a more robust and standardised process, within a transcriptomics laboratory, would lead to a GEP based signature which would be 
more likely to pass future independent validation studies (Figure 5). The technical variables should be considered as part of a comprehensive approach to RNA profiling studies requiring input from a multidisciplinary team (clinicians, statisticians, bioinformaticians, research scientists, health economists) both in the planning, execution and clinical impact assessment.
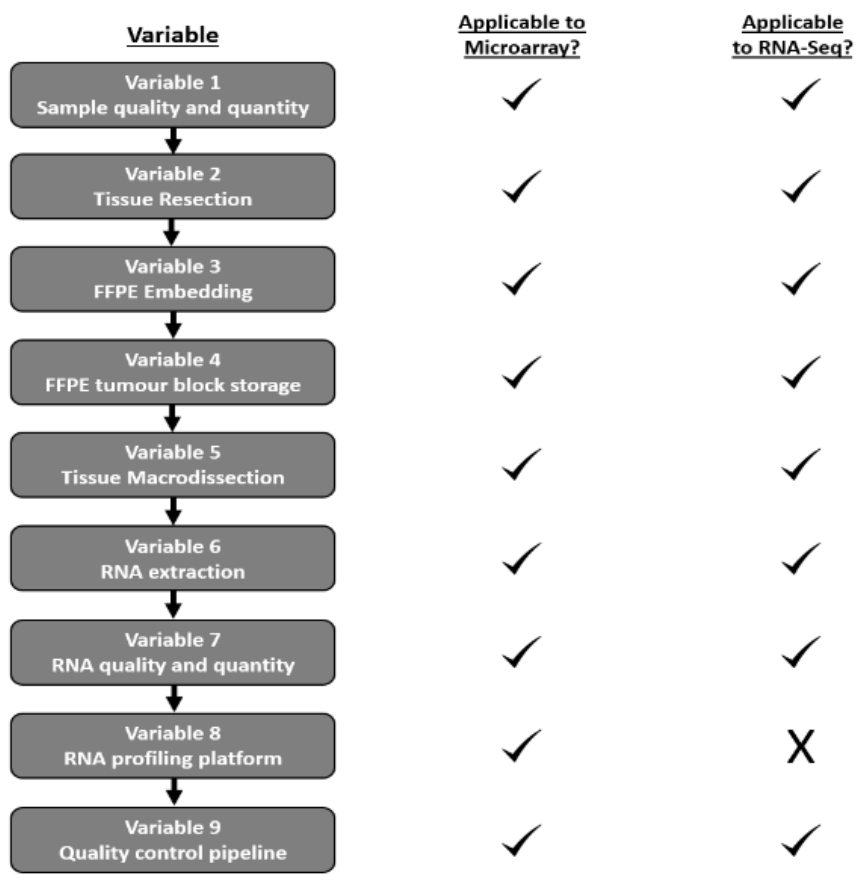

Figure 5: Flow diagram of the RNA profiling technical variables. This diagram depicts the nine different categories of technical variables that can affect results derived from high throughput RNA profiling technologies, such as microarray and RNA-Seq. It is recommended that investigators review the nine different categories and identify specific variables which are relevant to their particular RNA profiling studies.

Future development of in vitro diagnostic RNA profiling tests requires significant investment in technical and clinical validation by manufacturers, alongside development of an external quality assessment scheme. Economically, this investment needs to be balanced with considerations from the clinical diagnostics laboratory in purchasing and maintaining a RNA profiling platform, upskilling technical staff and considering RNA profiling test expenses 
which may limit the broad adoption of the test. Alternatively, RNA profiling, performed with consideration for the technical variables, could be used for GEP signature discovery before transferring to a more accessible technology such as a qPCR platform or miniarray for independent validation, test development and its commercialisation ${ }^{62}$. Irrespective of the approach, having a comprehensive understanding of RNA profiling technical variables, leading to consensus guidelines on how to identify and mitigate against them, will result in a more robust GEP signature suitable for clinical validation.

Acknowledgements: This study was supported by the Medical Research Council (MRC) and Cancer Research UK (CRUK) funded consortium 'Stratification in COloRectal cancer: from biology to Treatment prediction: S-CORT'

Conflict of Interest: The authors declare no competing conflict of interest.

Submission declaration: The manuscript has not been published or under review elsewhere and all authors approve publication of the final draft

Statement of author contributions: J.P.S. wrote the manuscript. S.R. T.M. and M.L. contributed and reviewed the manuscript. M.L. P.D. and M.S.T reviewed the manuscript and supervised the project. The authors have no conflicting financial interests. 


\section{$\underline{\text { References }}$}

1. Poston GJ, Tait D, O'Connell S, Bennett A, Berendse S, Guideline Development Group. Diagnosis and management of colorectal cancer: Summary of NICE guidance. BMJ. 2011;343:d6751. doi: 10.1136/bmj.d6751 [doi].

2. Edge S, Byrd D, Compton C, Fritz A, Greene F, Trotti A. Cancer staging manual. American Joint Committee on Cancer (AJCC).7th ed.New York: Springer. 2010.

3. Hari DM, Leung AM, Lee JH, et al. AJCC cancer staging manual 7th edition criteria for colon cancer: Do the complex modifications improve prognostic assessment? J Am Coll Surg. 2013;217(2):181-190. doi: 10.1016/j.jamcollsurg.2013.04.018 [doi].

4. Lech G, Slotwinski R, Slodkowski M, Krasnodebski IW. Colorectal cancer tumour markers and biomarkers: Recent therapeutic advances. World J Gastroenterol. 2016;22(5):1745-1755. doi: 10.3748/wjg.v22.i5.1745 [doi].

5. Masuda T, Ishikawa T, Mogushi K, et al. Overexpression of the S100A2 protein as a prognostic marker for patients with stage II and III colorectal cancer. Int J Oncol. 2016;48(3):975-982. doi: 10.3892/ijo.2016.3329 [doi].

6. Dalerba P, Sahoo D, Paik S, et al. CDX2 as a prognostic biomarker in stage II and stage III colon cancer. N Engl J Med. 2016;374(3):211-222. doi: 10.1056/NEJMoa1506597 [doi].

7. Mitrovic B, Schaeffer DF, Riddell RH, Kirsch R. Tumor budding in colorectal carcinoma: Time to take notice. Mod Pathol. 2012;25(10):1315-1325. doi: 10.1038/modpathol.2012.94 [doi]. 
8. Thirunavukarasu P, Talati C, Munjal S, Attwood K, Edge SB, Francescutti V. Effect of incorporation of pretreatment serum carcinoembryonic antigen levels into AJCC staging for colon cancer on 5-year survival. JAMA Surg. 2015;150(8):747-755. doi: 10.1001/jamasurg.2015.0871 [doi].

9. Rozek LS, Schmit SL, Greenson JK, et al. Tumor-infiltrating lymphocytes, crohn's-like lymphoid reaction, and survival from colorectal cancer. J Natl Cancer Inst. 2016;108(8):10.1093/jnci/djw027. Print 2016 Aug. doi: 10.1093/jnci/djw027 [doi].

10. Kern SE. Why your new cancer biomarker may never work: Recurrent patterns and remarkable diversity in biomarker failures. Cancer Res. 2012;72(23):6097-6101. doi: 10.1158/0008-5472.CAN-12-3232 [doi].

11. Voigt M, Braig F, Gothel M, et al. Functional dissection of the epidermal growth factor receptor epitopes targeted by panitumumab and cetuximab. Neoplasia. 2012;14(11):10231031.

12. Sorich MJ, Wiese MD, Rowland A, Kichenadasse G, McKinnon RA, Karapetis CS. Extended RAS mutations and anti-EGFR monoclonal antibody survival benefit in metastatic colorectal cancer: A meta-analysis of randomized, controlled trials. Ann Oncol. 2015;26(1):13-21. doi: 10.1093/annonc/mdu378 [doi].

13. Popovici V, Budinska E, Tejpar S, et al. Identification of a poor-prognosis BRAF-mutantlike population of patients with colon cancer. J Clin Oncol. 2012;30(12):1288-1295. doi: 10.1200/JCO.2011.39.5814 [doi].

14. Bertotti A, Migliardi G, Galimi F, et al. A molecularly annotated platform of patientderived xenografts ("xenopatients") identifies HER2 as an effective therapeutic target in 
cetuximab-resistant colorectal cancer. Cancer Discov. 2011;1(6):508-523. doi: 10.1158/21598290.CD-11-0109 [doi].

15. Misale S, Bozic I, Tong J, et al. Vertical suppression of the EGFR pathway prevents onset of resistance in colorectal cancers. Nat Commun. 2015;6:8305. doi: 10.1038/ncomms9305 [doi].

16. Medico E, Russo M, Picco G, et al. The molecular landscape of colorectal cancer cell lines unveils clinically actionable kinase targets. Nat Commun. 2015;6:7002. doi: 10.1038/ncomms8002 [doi].

17. Budinska E, Popovici V, Tejpar S, et al. Gene expression patterns unveil a new level of molecular heterogeneity in colorectal cancer. J Pathol. 2013;231(1):63-76. doi: 10.1002/path.4212 [doi].

18. Marisa L, de Reynies A, Duval A, et al. Gene expression classification of colon cancer into molecular subtypes: Characterization, validation, and prognostic value. PLoS Med. 2013;10(5):e1001453. doi: 10.1371/journal.pmed.1001453 [doi].

19. Roepman P, Schlicker A, Tabernero J, et al. Colorectal cancer intrinsic subtypes predict chemotherapy benefit, deficient mismatch repair and epithelial-to-mesenchymal transition. Int J Cancer. 2014;134(3):552-562. doi: 10.1002/ijc.28387 [doi].

20. De Sousa E Melo F, Wang X, Jansen M, et al. Poor-prognosis colon cancer is defined by a molecularly distinct subtype and develops from serrated precursor lesions. Nat Med. 2013;19(5):614-618. doi: 10.1038/nm.3174 [doi]. 
21. Sadanandam A, Lyssiotis CA, Homicsko K, et al. A colorectal cancer classification system that associates cellular phenotype and responses to therapy. Nat Med. 2013;19(5):619625. doi: $10.1038 / \mathrm{nm} .3175$ [doi].

22. Schlicker A, Beran G, Chresta CM, et al. Subtypes of primary colorectal tumors correlate with response to targeted treatment in colorectal cell lines. BMC Med Genomics. 2012;5:668794-5-66. doi: 10.1186/1755-8794-5-66 [doi].

23. Guinney J, Dienstmann R, Wang X, et al. The consensus molecular subtypes of colorectal cancer. Nat Med. 2015;21(11):1350-1356. doi: 10.1038/nm.3967 [doi].

24. Heberle H, Meirelles GV, da Silva FR, Telles GP, Minghim R. InteractiVenn: A webbased tool for the analysis of sets through venn diagrams. BMC Bioinformatics. 2015;16:169015-0611-3. doi: 10.1186/s12859-015-0611-3 [doi].

25. Youn HS, Kim TG, Kim MK, et al. Structural insights into the quaternary catalytic mechanism of hexameric human quinolinate phosphoribosyltransferase, a key enzyme in de novo NAD biosynthesis. Sci Rep. 2016;6:19681. doi: 10.1038/srep19681 [doi].

26. Sanz-Pamplona R, Berenguer A, Cordero D, et al. Clinical value of prognosis gene expression signatures in colorectal cancer: A systematic review. PLoS One. 2012;7(11):e48877. doi: 10.1371/journal.pone.0048877 [doi].

27. Ein-Dor L, Zuk O, Domany E. Thousands of samples are needed to generate a robust gene list for predicting outcome in cancer. Proc Natl Acad Sci U S A. 2006;103(15):59235928. doi: 0601231103 [pii]. 
28. McShane LM, Cavenagh MM, Lively TG, et al. Criteria for the use of omics-based predictors in clinical trials: Explanation and elaboration. BMC Med. 2013;11:220-7015-11220. doi: 10.1186/1741-7015-11-220 [doi].

29. Wang Y, Jatkoe T, Zhang Y, et al. Gene expression profiles and molecular markers to predict recurrence of dukes' B colon cancer. J Clin Oncol. 2004;22(9):1564-1571. doi: 10.1200/JCO.2004.08.186 [doi].

30. Bandres E, Malumbres R, Cubedo E, et al. A gene signature of 8 genes could identify the risk of recurrence and progression in dukes' B colon cancer patients. Oncol Rep. 2007;17(5):1089-1094.

31. Hao JM, Chen JZ, Sui HM, et al. A five-gene signature as a potential predictor of metastasis and survival in colorectal cancer. J Pathol. 2010;220(4):475-489. doi: 10.1002/path.2668 [doi].

32. Kalady MF, Dejulius K, Church JM, Lavery IC, Fazio VW, Ishwaran H. Gene signature is associated with early stage rectal cancer recurrence. J Am Coll Surg. 2010;211(2):187-195. doi: 10.1016/j.jamcollsurg.2010.03.035 [doi].

33. Pillaire MJ, Selves J, Gordien K, et al. A 'DNA replication' signature of progression and negative outcome in colorectal cancer. Oncogene. 2010;29(6):876-887. doi: 10.1038/onc.2009.378 [doi].

34. Schetter AJ, Nguyen GH, Bowman ED, et al. Association of inflammation-related and microRNA gene expression with cancer-specific mortality of colon adenocarcinoma. Clin Cancer Res. 2009;15(18):5878-5887. doi: 10.1158/1078-0432.CCR-09-0627 [doi]. 
35. Watanabe T, Kobunai T, Sakamoto E, et al. Gene expression signature for recurrence in stage III colorectal cancers. Cancer. 2009;115(2):283-292. doi: 10.1002/cncr.24023 [doi].

36. Eschrich S, Yang I, Bloom G, et al. Molecular staging for survival prediction of colorectal cancer patients. J Clin Oncol. 2005;23(15):3526-3535. doi: 23/15/3526 [pii].

37. Barrier A, Boelle PY, Roser F, et al. Stage II colon cancer prognosis prediction by tumor gene expression profiling. J Clin Oncol. 2006;24(29):4685-4691. doi: JCO.2005.05.0229 [pii].

38. Barrier A, Roser F, Boelle PY, et al. Prognosis of stage II colon cancer by non-neoplastic mucosa gene expression profiling. Oncogene. 2007;26(18):2642-2648. doi: 1210060 [pii].

39. Blum C, Graham A, Yousefzadeh M, et al. The expression ratio of Map7/B2M is prognostic for survival in patients with stage II colon cancer. Int J Oncol. 2008;33(3):579584.

40. Jorissen RN, Gibbs P, Christie M, et al. Metastasis-associated gene expression changes predict poor outcomes in patients with dukes stage B and C colorectal cancer. Clin Cancer Res. 2009;15(24):7642-7651. doi: 1078-0432.CCR-09-1431 [pii].

41. Wan YW, Qian Y, Rathnagiriswaran S, Castranova V, Guo NL. A breast cancer prognostic signature predicts clinical outcomes in multiple tumor types. Oncol Rep. 2010;24(2):489-494.

42. Smith JJ, Deane NG, Wu F, et al. Experimentally derived metastasis gene expression profile predicts recurrence and death in patients with colon cancer. Gastroenterology. 2010;138(3):958-968. doi: 10.1053/j.gastro.2009.11.005 [doi]. 
43. Mettu RK, Wan YW, Habermann JK, Ried T, Guo NL. A 12-gene genomic instability signature predicts clinical outcomes in multiple cancer types. Int J Biol Markers. 2010;25(4):219-228. doi: EEBE41CB-C3E2-410C-8914-215C4B861868 [pii].

44. Hong Y, Downey T, Eu KW, Koh PK, Cheah PY. A 'metastasis-prone' signature for early-stage mismatch-repair proficient sporadic colorectal cancer patients and its implications for possible therapeutics. Clin Exp Metastasis. 2010;27(2):83-90. doi: 10.1007/s10585-0109305-4 [doi].

45. Schee K, Lorenz S, Worren MM, et al. Deep sequencing the MicroRNA transcriptome in colorectal cancer. PLoS One. 2013;8(6):e66165. doi: 10.1371/journal.pone.0066165 [doi].

46. Bertucci F, Salas S, Eysteries S, et al. Gene expression profiling of colon cancer by DNA microarrays and correlation with histoclinical parameters. Oncogene. 2004;23(7):1377-1391. doi: 10.1038/sj.onc.1207262 [doi].

47. Arango D, Laiho P, Kokko A, et al. Gene-expression profiling predicts recurrence in dukes' C colorectal cancer. Gastroenterology. 2005;129(3):874-884. doi: S00165085(05)01354-5 [pii].

48. D'Arrigo A, Belluco C, Ambrosi A, et al. Metastatic transcriptional pattern revealed by gene expression profiling in primary colorectal carcinoma. Int J Cancer. 2005;115(2):256262. doi: 10.1002/ijc.20883 [doi].

49. Yamasaki M, Takemasa I, Komori T, et al. The gene expression profile represents the molecular nature of liver metastasis in colorectal cancer. Int J Oncol. 2007;30(1):129-138. 
50. Lin YH, Friederichs J, Black MA, et al. Multiple gene expression classifiers from different array platforms predict poor prognosis of colorectal cancer. Clin Cancer Res. 2007;13(2 Pt 1):498-507. doi: 13/2/498 [pii].

51. Anjomshoaa A, Lin YH, Black MA, et al. Reduced expression of a gene proliferation signature is associated with enhanced malignancy in colon cancer. $\mathrm{Br} J$ Cancer. 2008;99(6):966-973.

52. Staub E, Groene J, Heinze M, et al. An expression module of WIPF1-coexpressed genes identifies patients with favorable prognosis in three tumor types. J Mol Med (Berl). 2009;87(6):633-644. doi: 10.1007/s00109-009-0467-y [doi].

53. Matsuyama T, Ishikawa T, Mogushi K, et al. MUC12 mRNA expression is an independent marker of prognosis in stage II and stage III colorectal cancer. Int J Cancer. 2010;127(10):2292-2299. doi: 10.1002/ijc.25256 [doi].

54. Schepeler T, Reinert JT, Ostenfeld MS, et al. Diagnostic and prognostic microRNAs in stage II colon cancer. Cancer Res. 2008;68(15):6416-6424. doi: 10.1158/0008-5472.CAN07-6110 [doi].

55. Nguyen MN, Choi TG, Nguyen DT, et al. CRC-113 gene expression signature for predicting prognosis in patients with colorectal cancer. Oncotarget. 2015;6(31):31674-31692. doi: 10.18632/oncotarget.5183 [doi].

56. Fehlker M, Huska MR, Jons T, Andrade-Navarro MA, Kemmner W. Concerted downregulation of immune-system related genes predicts metastasis in colorectal carcinoma. $B M C$ Cancer. 2014;14:64-2407-14-64. doi: 10.1186/1471-2407-14-64 [doi]. 
57. Xue Y, Ma G, Gu D, et al. Genome-wide analysis of long noncoding RNA signature in human colorectal cancer. Gene. 2015;556(2):227-234. doi: 10.1016/j.gene.2014.11.060 [doi].

58. Agesen TH, Sveen A, Merok MA, et al. ColoGuideEx: A robust gene classifier specific for stage II colorectal cancer prognosis. Gut. 2012;61(11):1560-1567. doi: gutjnl-2011301179 [pii].

59. Sveen A, Agesen TH, Nesbakken A, et al. ColoGuidePro: A prognostic 7-gene expression signature for stage III colorectal cancer patients. Clin Cancer Res. 2012;18(21):6001-6010. doi: 10.1158/1078-0432.CCR-11-3302 [doi].

60. Lenehan PF, Boardman LA, Riegert-Johnson D, et al. Generation and external validation of a tumor-derived 5-gene prognostic signature for recurrence of lymph node-negative, invasive colorectal carcinoma. Cancer. 2012;118(21):5234-5244. doi: 10.1002/cncr.27628 [doi].

61. Kennedy RD, Bylesjo M, Kerr P, et al. Development and independent validation of a prognostic assay for stage II colon cancer using formalin-fixed paraffin-embedded tissue. $J$ Clin Oncol. 2011;29(35):4620-4626. doi: 10.1200/JCO.2011.35.4498 [doi].

62. Van Laar RK. An online gene expression assay for determining adjuvant therapy eligibility in patients with stage 2 or 3 colon cancer. Br J Cancer. 2010;103(12):1852-1857. doi: 10.1038/sj.bjc.6605970 [doi].

63. Ciarloni L, Hosseinian S, Monnier-Benoit S, et al. Discovery of a 29-gene panel in peripheral blood mononuclear cells for the detection of colorectal cancer and adenomas using high throughput real-time PCR. PLoS One. 2015;10(4):e0123904. doi: 10.1371/journal.pone.0123904 [doi]. 
64. Blow N. Tissue preparation: Tissue issues. Nature. 2007;448(7156):959-963. doi: 448959a [pii].

65. Barrier A, Lemoine A, Boelle PY, et al. Colon cancer prognosis prediction by gene expression profiling. Oncogene. 2005;24(40):6155-6164. doi: 1208984 [pii].

66. O'Connell MJ, Lavery I, Yothers G, et al. Relationship between tumor gene expression and recurrence in four independent studies of patients with stage II/III colon cancer treated with surgery alone or surgery plus adjuvant fluorouracil plus leucovorin. J Clin Oncol. 2010;28(25):3937-3944. doi: 10.1200/JCO.2010.28.9538 [doi].

67. Jiang Y, Casey G, Lavery IC, et al. Development of a clinically feasible molecular assay to predict recurrence of stage II colon cancer. J Mol Diagn. 2008;10(4):346-354. doi: 10.2353/jmoldx.2008.080011 [doi].

68. Andersen CL, Christensen LL, Thorsen K, et al. Dysregulation of the transcription factors SOX4, CBFB and SMARCC1 correlates with outcome of colorectal cancer. Br J Cancer. 2009;100(3):511-523. doi: 10.1038/sj.bjc.6604884 [doi].

69. Watanabe T, Kobunai T, Yamamoto Y, et al. Prediction of liver metastasis after colorectal cancer using reverse transcription-polymerase chain reaction analysis of 10 genes. Eur J Cancer. 2010;46(11):2119-2126. doi: 10.1016/j.ejca.2010.04.019 [doi].

70. Salazar R, Roepman P, Capella G, et al. Gene expression signature to improve prognosis prediction of stage II and III colorectal cancer. J Clin Oncol. 2011;29(1):17-24. doi: 10.1200/JCO.2010.30.1077 [doi]. 
71. Peng J, Wang Z, Chen W, et al. Integration of genetic signature and TNM staging system for predicting the relapse of locally advanced colorectal cancer. Int J Colorectal Dis. 2010;25(11):1277-1285. doi: 10.1007/s00384-010-1043-1 [doi].

72. Zhang JX, Song W, Chen ZH, et al. Prognostic and predictive value of a microRNA signature in stage II colon cancer: A microRNA expression analysis. Lancet Oncol. 2013;14(13):1295-1306. doi: 10.1016/S1470-2045(13)70491-1 [doi].

73. Chen D, Sun Q, Cheng X, et al. Genome-wide analysis of long noncoding RNA (lncRNA) expression in colorectal cancer tissues from patients with liver metastasis. Cancer Med. 2016;5(7):1629-1639. doi: 10.1002/cam4.738 [doi].

74. Ghadimi BM, Grade M, Difilippantonio MJ, et al. Effectiveness of gene expression profiling for response prediction of rectal adenocarcinomas to preoperative chemoradiotherapy. J Clin Oncol. 2005;23(9):1826-1838. doi: 23/9/1826 [pii].

75. Liersch T, Grade M, Gaedcke J, et al. Preoperative chemoradiotherapy in locally advanced rectal cancer: Correlation of a gene expression-based response signature with recurrence. Cancer Genet Cytogenet. 2009;190(2):57-65. doi:

10.1016/j.cancergencyto.2008.11.011 [doi].

76. Estevez-Garcia P, Rivera F, Molina-Pinelo S, et al. Gene expression profile predictive of response to chemotherapy in metastatic colorectal cancer. Oncotarget. 2015;6(8):6151-6159. doi: 3152 [pii].

77. Del Rio M, Molina F, Bascoul-Mollevi C, et al. Gene expression signature in advanced colorectal cancer patients select drugs and response for the use of leucovorin, fluorouracil, and irinotecan. J Clin Oncol. 2007;25(7):773-780. doi: 25/7/773 [pii]. 
78. Molina-Pinelo S, Carnero A, Rivera F, et al. MiR-107 and miR-99a-3p predict chemotherapy response in patients with advanced colorectal cancer. BMC Cancer. 2014;14:656-2407-14-656. doi: 10.1186/1471-2407-14-656 [doi].

79. Lee JR, Kwon $\mathrm{CH}$, Choi Y, et al. Transcriptome analysis of paired primary colorectal carcinoma and liver metastases reveals fusion transcripts and similar gene expression profiles in primary carcinoma and liver metastases. BMC Cancer. 2016;16:539-016-2596-3. doi: 10.1186/s12885-016-2596-3 [doi].

80. Xu L, Ziegelbauer J, Wang R, et al. Distinct profiles for mitochondrial t-RNAs and small nucleolar RNAs in locally invasive and metastatic colorectal cancer. Clin Cancer Res. 2016;22(3):773-784. doi: 10.1158/1078-0432.CCR-15-0737 [doi].

81. Kim SK, Kim SY, Kim JH, et al. A nineteen gene-based risk score classifier predicts prognosis of colorectal cancer patients. Mol Oncol. 2014;8(8):1653-1666. doi: 10.1016/j.molonc.2014.06.016 [doi].

82. Wu Y, Wang X, Wu F, et al. Transcriptome profiling of the cancer, adjacent non-tumor and distant normal tissues from a colorectal cancer patient by deep sequencing. PLoS One. 2012;7(8):e41001. doi: 10.1371/journal.pone.0041001 [doi].

83. Lawler M, Kaplan R, Wilson RH, Maughan T, S-CORT Consortium. Changing the paradigm-multistage multiarm randomized trials and stratified cancer medicine. Oncologist. 2015;20(8):849-851. doi: 10.1634/theoncologist.2015-0014 [doi].

84. Dunne PD, McArt DG, Bradley CA, et al. Challenging the cancer molecular stratification dogma: Intratumoral heterogeneity undermines consensus molecular subtypes and potential 
diagnostic value in colorectal cancer. Clin Cancer Res. 2016;22(16):4095-4104. doi: 10.1158/1078-0432.CCR-16-0032 [doi].

85. Waltz E. Pricey cancer genome project struggles with sample shortage. Nat Med. 2007;13(4):391. doi: nm0407-391 [pii].

86. David KA, Unger FT, Uhlig P, et al. Surgical procedures and postsurgical tissue processing significantly affect expression of genes and EGFR-pathway proteins in colorectal cancer tissue. Oncotarget. 2014;5(22):11017-11028. doi: 10.18632/oncotarget.2669 [doi].

87. Bao WG, Zhang X, Zhang JG, et al. Biobanking of fresh-frozen human colon tissues: Impact of tissue ex-vivo ischemia times and storage periods on RNA quality. Ann Surg Oncol. 2013;20(5):1737-1744. doi: 10.1245/s10434-012-2440-1 [doi].

88. Lee SM, Schelcher C, Thasler R, Schiergens TS, Thasler WE. Pre-analytical determination of the effect of extended warm or cold ischaemia on RNA stability in the human ileum mucosa. PLoS One. 2015;10(9):e0138214. doi: 10.1371/journal.pone.0138214 [doi].

89. Guerrera F, Tabb $\tilde{A}^{2} \mathrm{~F}$, Bessone L, et al. The influence of tissue ischemia time on RNA integrity and patient-derived xenografts (PDX) engraftment rate in a non-small cell lung cancer (NSCLC) biobank. PLoS One. 2016;11(1):e0145100.

doi:10.1371/journal.pone.0145100. doi: 10.1371/journal.pone.0145100 [doi].

90. Miyatake Y, Ikeda H, Michimata R, et al. Differential modulation of gene expression among rat tissues with warm ischemia. Exp Mol Pathol. 2004;77(3):222-230. doi: S00144800(04)00068-1 [pii]. 
91. Lange N, Unger FT, Schoppler M, Pursche K, Juhl H, David KA. Identification and validation of a potential marker of tissue quality using gene expression analysis of human colorectal tissue. PLoS One. 2015;10(7):e0133987. doi: 10.1371/journal.pone.0133987 [doi].

92. Staff S, Kujala P, Karhu R, et al. Preservation of nucleic acids and tissue morphology in paraffin-embedded clinical samples: Comparison of five molecular fixatives. J Clin Pathol. 2013;66(9):807-810. doi: 10.1136/jclinpath-2012-201283 [doi].

93. Howat WJ, Wilson BA. Tissue fixation and the effect of molecular fixatives on downstream staining procedures. Methods. 2014;70(1):12-19. doi:

10.1016/j.ymeth.2014.01.022 [doi].

94. Srinivasan M, Sedmak D, Jewell S. Effect of fixatives and tissue processing on the content and integrity of nucleic acids. Am J Pathol. 2002;161(6):1961-1971. doi: S00029440(10)64472-0 [pii].

95. Scicchitano MS, Dalmas DA, Bertiaux MA, et al. Preliminary comparison of quantity, quality, and microarray performance of RNA extracted from formalin-fixed, paraffinembedded, and unfixed frozen tissue samples. J Histochem Cytochem. 2006;54(11):12291237. doi: jhc.6A6999.2006 [pii].

96. Wong SQ, Li J, Tan AY, et al. Sequence artefacts in a prospective series of formalinfixed tumours tested for mutations in hotspot regions by massively parallel sequencing. $B M C$ Med Genomics. 2014;7:23-8794-7-23. doi: 10.1186/1755-8794-7-23 [doi].

97. Greytak SR, Engel KB, Bass BP, Moore HM. Accuracy of molecular data generated with FFPE biospecimens: Lessons from the literature. Cancer Res. 2015;75(8):1541-1547. doi: 10.1158/0008-5472.CAN-14-2378 [doi]. 
98. von Ahlfen S, Missel A, Bendrat K, Schlumpberger M. Determinants of RNA quality from FFPE samples. PLoS One. 2007;2(12):e1261. doi: 10.1371/journal.pone.0001261 [doi].

99. Abdueva D, Wing M, Schaub B, Triche T, Davicioni E. Quantitative expression profiling in formalin-fixed paraffin-embedded samples by affymetrix microarrays. J Mol Diagn. 2010;12(4):409-417. doi: 10.2353/jmoldx.2010.090155 [doi].

100. Hamilton PW, Wang Y, Boyd C, et al. Automated tumor analysis for molecular profiling in lung cancer. Oncotarget. 2015;6(29):27938-27952. doi: 10.18632/oncotarget.4391 [doi].

101. Muley TR, Herth FJ, Schnabel PA, Dienemann H, Meister M. From tissue to molecular phenotyping: Pre-analytical requirements heidelberg experience. Transl Lung Cancer Res. 2012;1(2):111-121. doi: 10.3978/j.issn.2218-6751.2011.12.07 [doi].

102. Viray H, Li K, Long TA, et al. A prospective, multi-institutional diagnostic trial to determine pathologist accuracy in estimation of percentage of malignant cells. Arch Pathol Lab Med. 2013;137(11):1545-1549. doi: 10.5858/arpa.2012-0561-CP [doi].

103. Cleator SJ, Powles TJ, Dexter T, et al. The effect of the stromal component of breast tumours on prediction of clinical outcome using gene expression microarray analysis. Breast Cancer Res. 2006;8(3):R32. doi: bcr1506 [pii].

104. Roepman P, Schuurman A, Delahaye LJ, Witteveen AT, Floore AN, Glas AM. A gene expression profile for detection of sufficient tumour cells in breast tumour tissue: Microarray diagnosis eligibility. BMC Med Genomics. 2009;2:52-8794-2-52. doi: 10.1186/1755-8794-252 [doi]. 
105. Becht E, de Reynies A, Giraldo NA, et al. Immune and stromal classification of colorectal cancer is associated with molecular subtypes and relevant for precision immunotherapy. Clin Cancer Res. 2016;22(16):4057-4066. doi: 10.1158/1078-0432.CCR-152879 [doi].

106. Masuda N, Ohnishi T, Kawamoto S, Monden M, Okubo K. Analysis of chemical modification of RNA from formalin-fixed samples and optimization of molecular biology applications for such samples. Nucleic Acids Res. 1999;27(22):4436-4443. doi: gkc634 [pii].

107. Hall JS, Taylor J, Valentine HR, et al. Enhanced stability of microRNA expression facilitates classification of FFPE tumour samples exhibiting near total mRNA degradation. $\mathrm{Br}$ J Cancer. 2012;107(4):684-694. doi: 10.1038/bjc.2012.294 [doi].

108. Doleshal M, Magotra AA, Choudhury B, Cannon BD, Labourier E, Szafranska AE. Evaluation and validation of total RNA extraction methods for microRNA expression analyses in formalin-fixed, paraffin-embedded tissues. J Mol Diagn. 2008;10(3):203-211. doi: 10.2353/jmoldx.2008.070153 [doi].

109. Desjardins P, Conklin D. NanoDrop microvolume quantitation of nucleic acids. J Vis Exp. 2010;(45). pii: 2565. doi(45):10.3791/2565. doi: 10.3791/2565 [doi].

110. Aranda R,4th, Dineen SM, Craig RL, Guerrieri RA, Robertson JM. Comparison and evaluation of RNA quantification methods using viral, prokaryotic, and eukaryotic RNA over a 10(4) concentration range. Anal Biochem. 2009;387(1):122-127. doi:

10.1016/j.ab.2009.01.003 [doi]. 
111. Thorsteinsson M, Kirkeby LT, Hansen R, et al. Gene expression profiles in stages II and III colon cancers: Application of a 128-gene signature. Int J Colorectal Dis.

2012;27(12):1579-1586. doi: 10.1007/s00384-012-1517-4 [doi].

112. Takano EA, Mikeska T, Dobrovic A, Byrne DJ, Fox SB. A multiplex endpoint RT-PCR assay for quality assessment of RNA extracted from formalin-fixed paraffin-embedded tissues. BMC Biotechnol. 2010;10:89-6750-10-89. doi: 10.1186/1472-6750-10-89 [doi].

113. 2016-NCIBestPractices.pdf. http://biospecimens.cancer.gov/bestpractices/2016NCIBestPractices.pdf. Accessed 8/23/2016, 2016.

114. Opitz L, Salinas-Riester G, Grade M, et al. Impact of RNA degradation on gene expression profiling. BMC Med Genomics. 2010;3:36-8794-3-36. doi: 10.1186/1755-8794-336 [doi].

115. Leek JT, Scharpf RB, Bravo HC, et al. Tackling the widespread and critical impact of batch effects in high-throughput data. Nat Rev Genet. 2010;11(10):733-739. doi: $10.1038 / \operatorname{nrg} 2825$ [doi].

116. Hsu FH, Serpedin E, Hsiao TH, Bishop AJ, Dougherty ER, Chen Y. Reducing confounding and suppression effects in TCGA data: An integrated analysis of chemotherapy response in ovarian cancer. BMC Genomics. 2012;13 Suppl 6:S13-2164-13-S6-S13. Epub 2012 Oct 26. doi: 10.1186/1471-2164-13-S6-S13 [doi].

117. Baker SC, Bauer SR, Beyer RP, et al. The external RNA controls consortium: A progress report. Nat Methods. 2005;2(10):731-734. doi: nmeth1005-731 [pii]. 
118. Tong W, Lucas AB, Shippy R, et al. Evaluation of external RNA controls for the assessment of microarray performance. Nat Biotechnol. 2006;24(9):1132-1139. doi: nbt1237 [pii].

119. Jiang L, Schlesinger F, Davis CA, et al. Synthetic spike-in standards for RNA-seq experiments. Genome Res. 2011;21(9):1543-1551. doi: 10.1101/gr.121095.111 [doi].

120. Xu J, Su Z, Hong H, et al. Cross-platform ultradeep transcriptomic profiling of human reference RNA samples by RNA-seq. Sci Data. 2014;1:140020. doi: 10.1038/sdata.2014.20 [doi].

121. Clark MB, Mercer TR, Bussotti G, et al. Quantitative gene profiling of long noncoding RNAs with targeted RNA sequencing. Nat Methods. 2015;12(4):339-342. doi: 10.1038/nmeth.3321 [doi].

122. Dalma-Weiszhausz DD, Warrington J, Tanimoto EY, Miyada CG. The affymetrix GeneChip platform: An overview. Methods Enzymol. 2006;410:3-28. doi: S00766879(06)10001-4 [pii].

123. Salto-Tellez M, Gonzalez de Castro D. Next-generation sequencing: A change of paradigm in molecular diagnostic validation. J Pathol. 2014;234(1):5-10. doi: 10.1002/path.4365 [doi].

124. Durinck S. Pre-processing of microarray data and analysis of differential expression. Bioinformatics: Data, Sequence Analysis and Evolution. 2008:89-110.

125. Cordero F, Botta M, Calogero RA. Microarray data analysis and mining approaches. Brief Funct Genomic Proteomic. 2007;6(4):265-281. doi: 10.1093/bfgp/elm034 [doi]. 
126. Shakya K, Ruskin HJ, Kerr G, Crane M, Becker J. Comparison of microarray preprocessing methods. Adv Exp Med Biol. 2010;680:139-147. doi: 10.1007/978-1-44195913-3_16 [doi].

127. McCall MN, Almudevar A. Affymetrix GeneChip microarray preprocessing for multivariate analyses. Brief Bioinform. 2012;13(5):536-546. doi: 10.1093/bib/bbr072 [doi].

128. Glas AM, Floore A, Delahaye LJ, et al. Converting a breast cancer microarray signature into a high-throughput diagnostic test. BMC Genomics. 2006;7:278. doi: 1471-2164-7-278 [pii].

129. Mulligan JM, Hill LA, Deharo S, et al. Identification and validation of an anthracycline/cyclophosphamide-based chemotherapy response assay in breast cancer. $J$ Natl Cancer Inst. 2014;106(1):djt335. doi: 10.1093/jnci/djt335 [doi].

130. van Laar R, Flinchum R, Brown N, et al. Translating a gene expression signature for multiple myeloma prognosis into a robust high-throughput assay for clinical use. $B M C M e d$ Genomics. 2014;7:25-8794-7-25. doi: 10.1186/1755-8794-7-25 [doi].

131. Niedzwiecki D, Frankel WL, Venook AP, et al. Association between results of a gene expression signature assay and recurrence-free interval in patients with stage II colon cancer in cancer and leukemia group B 9581 (alliance). J Clin Oncol. 2016;34(25):3047-3053. doi: 10.1200/JCO.2015.65.4699 [doi].

132. Zhao X, Rodland EA, Sorlie T, et al. Systematic assessment of prognostic gene signatures for breast cancer shows distinct influence of time and ER status. BMC Cancer. 2014;14:211-2407-14-211. doi: 10.1186/1471-2407-14-211 [doi]. 
133. Kim SM, Leem SH, Chu IS, et al. Sixty-five gene-based risk score classifier predicts overall survival in hepatocellular carcinoma. Hepatology. 2012;55(5):1443-1452. doi: 10.1002/hep.24813 [doi].

134. Metzeler KH, Hummel M, Bloomfield CD, et al. An 86-probe-set gene-expression signature predicts survival in cytogenetically normal acute myeloid leukemia. Blood. 2008;112(10):4193-4201. doi: 10.1182/blood-2008-02-134411 [doi].

135. Scott MG, Ashwood ER, Annesley TM, Leonard DG, Burgess MC. FDA oversight of laboratory-developed tests: Is it necessary, and how would it impact clinical laboratories? Clin Chem. 2013;59(7):1017-1022. doi: 10.1373/clinchem.2013.204958 [doi].

136. The public health evidence for FDA oversighht of laboratory developed tests: 20 case studies - UCM472777.pdf.

http://www.fda.gov/downloads/AboutFDA/ReportsManualsForms/Reports/UCM472777.pdf. Accessed 10/12/2016, 2016.

137. Marzinke MA, Clarke W. Laboratory developed tests in the clinical laboratory: Challenges for implementation. Bioanalysis. 2015;7(15):1817-1820. doi: 10.4155/bio.15.109 [doi].

138. Torlakovic EE, Nielsen S, Vyberg M, Taylor CR. Getting controls under control: The time is now for immunohistochemistry. J Clin Pathol. 2015;68(11):879-882. doi: 10.1136/jclinpath-2014-202705 [doi]. 